\title{
Increasing the antioxidant capability via the synergistic effect of coupling diphenylamine with sterically hindered phenol
}

Article

Accepted Version

Higgins, C. L., Filip, S. V., Afsar, A. and Hayes, W. (2019) Increasing the antioxidant capability via the synergistic effect of coupling diphenylamine with sterically hindered phenol. Tetrahedron, 75 (51). 130759. ISSN 0040-4020 doi: https://doi.org/10.1016/j.tet.2019.130759 Available at https://centaur.reading.ac.uk/87157/

It is advisable to refer to the publisher's version if you intend to cite from the work. See Guidance on citing.

To link to this article DOI: http://dx.doi.org/10.1016/j.tet.2019.130759

Publisher: Elsevier

All outputs in CentAUR are protected by Intellectual Property Rights law, including copyright law. Copyright and IPR is retained by the creators or other copyright holders. Terms and conditions for use of this material are defined in the End User Agreement. 


\section{CentAUR}

Central Archive at the University of Reading

Reading's research outputs online 


\section{Increasing the antioxidant capability via the synergistic effect of coupling diphenylamine with sterically hindered phenol}

Clare L. Higgins ${ }^{1}$, Sorin V. Filip ${ }^{2}$, Ashfaq Afsar ${ }^{1}$ and Wayne Hayes ${ }^{1 *}$

${ }^{1}$ Department of Chemistry, University of Reading, Whiteknights, Reading, RG6 6AD, UK.

${ }^{2}$ BP Formulated Products Technology, Research \& Innovation, Pangbourne, UK.

* Corresponding author. Tel.: +44118 378 6491, Fax: +44 1183786331

Email address:w.c.hayes@reading.ac.uk

Abstract: A series of novel diphenylamine-phenol antioxidants were synthesised that combined the two antioxidant types into a single molecule. These antioxidants were then functionalised with alkyl chains to aid their solubility in hydrocarbon media. As part of a structure-activity study, diphenylamine derivatives were also generated bearing carboxylic acid functionalities in either the ortho, meta or para position with respect to the secondary amine. Methyl or ethyl spacers were also incorporated between the carboxylic acid and the aromatic ring. The antioxidant ability of the diphenylamine-phenols was evaluated using Differential Scanning Calorimetry (DSC) and compared to commercially available antioxidants Irganox L135 and Irganox L57 both as individual components and when blended together. The diphenylamine-phenol antioxidant with an ethyl spacer between the diphenylamine and carboxylic acid in the meta position with respect to the secondary amine functionality showed an impressive oxidation induction time of ca. 24 minutes in direct comparison with the blend of Irganox L135 and Irganox L57 (ca. 16 minutes).

Keywords: Synergism, Dual functional antioxidants, Diphenylamines, Phenols, Oxidative Stability Studies

\section{Introduction}


Oxidative degradation of hydrocarbon materials such as oils, fuels, biofuels, and polymers is a common problem in several industrial processes. [1-4] In particular, when an automotive lubricant or a lubricant for any mechanical system is being used, this oxidizes more easily, depending on its type (paraffinic, naphthenic, or aromatic), to form harmful species which can shorten its service life and ultimately damage the physical integrity of the machinery. [5-6] Among several possibilities to reduce or prevent the speed of lubricant oxidation, antioxidants are the key additives incorporated by chemical industries in their lubricant formulations to protect oils from oxidative degradation and prolong service life. [7-11] Phenols and diphenylamines constitute the bulk of radical trapping antioxidants that are incorporated into lubricant formulations because of their high inherent reactivities toward peroxyl radicals and the persistence of the resultant radicals toward both $\mathrm{O}_{2}$ and non-radical species. [12-14] Phenols, especially sterically hindered phenols, are environmentally friendly and have low toxicity which make them prevalent in automotive lubricating oils but are restricted from high temperature applications because of their poor thermal stability and liability to failure owing to photochromatic effect under severe conditions. [15-18] In contrast, diphenylamines have better performance at elevated temperatures but the antioxidant performance still needs to be improved. [19-21] Complying with these limitations, there is an increasing demand to meet strict emission legislation whilst enhancing fuel economy thus forcing the petrochemical industry to develop highly effective antioxidants for used in fuels. [22] Therefore, in order to meet these demands upon the properties of lubricating oils, improving via the synergistic means the performance of commercial antioxidants using sulfur-, phosphorus- and heavy metal-free compounds is desirable. [23] The term 'synergism' in this case refers to the cooperative action of two or more additive species in such a way that the total antioxidant effect is greater than the sum of the individual effects taken independently. [24] It is wellknown, particularly in some lubricant formulations that, sterically hindered phenols and 
diphenylamines, when added to the oil simultaneously can remarkably enhance the antioxidant efficiency due to their intermolecular synergistic effect. [25-28] These exciting results prompted us to design a series of antioxidants that combines the sterically hindered phenol and diphenylamine moieties into a single structure to form a bifunctional antioxidant. The benefits of this approach are that increasing molecular weight of the antioxidant could also improve the thermal stability at elevated temperatures and could reduce the amount of additives used in formulations. [29] Although considerable efforts have been devoted to investigating and improving the individual antioxidant capabilities of hindered phenols and diphenylamines through structural variations [30-33], only a few examples have been reported that detail the inclusion of both functional groups within the same compound. [3435] Recently Liu et al. [36] designed a new type of antioxidant which combined the two antioxidant types into a one molecule via Schiff base (Figure 1). These Schiff base antioxidants bridged phenolic diphenylamine antioxidants (SPD1 and SPD2) exhibited better thermal stability and antioxidant efficiency than the commonly used commercial antioxidants. Inspired by this study, we report the design and synthesis of a series of novel mixed diphenylamine-phenol antioxidants that combine the two traditional antioxidants into one molecule in addition to alkyl chains to gain better solubility. To further investigate the structure-activity relationship, diphenylamine derivatives that possess carboxylic acid functionalities in either ortho, meta or para position with respect to the secondary amine and methyl or ethyl spacers were incorporated between the carboxylic acid and the aromatic ring. Their antioxidant ability was examined using pressurised differential scanning calorimetry (PDSC) and the antioxidant performance compared to the current industry antioxidants Irganox L135 and Irganox L57 (Figure 2) both as individual components and as a synergistic blend together.

\section{Results and discussion}




\subsection{Synthesis}

The palladium catalysed Buchwald-Hartwig amination [37-39] was used to synthesise the ester derivative 3 (see SI Scheme S1). [40-41] After base catalysed ester hydrolysis, the acid derivative of diphenylamine 4 and 3-(3,5 di tert-butyl 4 hydroxyphenyl)propionic acid $\mathbf{7}$ were attached to the first generation hydroxyl linker 6. [29] Two different synthetic procedures were targeted, both utilising $N, N^{\prime}$ dicyclohexylcarbodiimide (DCC) mediated coupling. The first approach, shown in Scheme 1, involved a statistical reaction whereby 4, the hindered phenol 7 and 6 were reacted in a ratio of 1:1:1. The statistical reaction yielded successfully the desired compound 8 in ca. 30\%, however, the competing by-products 9 and 10, shown in Figure 3, were isolated in a yield of ca. 30\% each, respectively. To improve production of $\mathbf{8}$, an alternative synthetic methodology was utilised where a mono-phenolic hydroxyl linker $\mathbf{5}$ was synthesised from the reaction of 7 with a single hydroxyl functionality of 6 . Subsequently, the reaction of 5 with diphenylamine $\mathbf{4}$ via DCC mediated coupling (Scheme 2) afforded the desired mixed amine-phenol 8 with an improved yield of ca. $70 \%$. To investigate the structureactivity relationships of the novel mixed amine-phenol, para-substituted derivatives of mixed amino-phenol 11 and bis(diphenylamine) 13 (see Series 1, Table 1) were prepared by reacting 2-(4-(phenylamino)phenyl)acetic acid 12 with 5 and 6 following the above synthetic methodology (see SI Scheme S2). To further refine the structure of the mixed amine-phenols, an additional alkyl chain was introduced in Series 2 by employing 4-butylaniline 16 as the starting material. The most effective position to link the diphenylamine to the hydroxyl linker was also investigated and ortho, meta and para carboxylic acid substituted diphenylamines were synthesised via the Buchwald-Hartwig amination [37-39] methodology previously described. The esters 14 (ortho), 17 (meta) and 19 (para) were generated, followed by base hydrolysis to afford the carboxylic acids 21-23 in good yields (>80\%) as shown in Scheme 3 . [42-45] Successful coupling of 4-butylaniline 16 to methyl 3-bromobenzoate 18 and methyl 
4-bromobenzoate 20 was achieved to yield the meta and para diphenylamines 17 and $\mathbf{1 9}$, respectively (isolated yields ca. 50\%). However, the synthesis of 14, using methyl 2bromobenzoate 15 , proved to be more challenging and a low yield of only ca. $25 \%$ was achieved. In an attempt to improve the yield, $\mathrm{K}_{2} \mathrm{CO}_{3}$ was used as a base for the BuchwaldHartwig amination reaction and a significant improvement in the isolated yield $(53 \%)$ was observed. With the (ortho, meta and para)-substituted diphenylamines derivatives 21-23 and mono-phenolic hydroxyl linker 5 in hand, mixed amine-phenols 24-26 were prepared successfully employing the same methodology described to generate Series 1. In addition, the bis(diphenylamines) 27-29 were also generated from the reaction of 21-23 with first generation diol linker 6 (see Series 2, Table 2). To conclude the series, the inclusion of a methyl or ethyl spacer between the carboxylic acid and aromatic ring was also investigated. The esters 30 (meta- $\left.\mathrm{CH}_{2} \mathrm{CH}_{2}-\right)$, 32 (para $-\mathrm{CH}_{2}$ ) and $\mathbf{3 4}$ (para $-\mathrm{CH}_{2} \mathrm{CH}_{2}$ ) were generated first by coupling of 16 to 31,33 and 35 respectively, followed by ester hydrolysis to yield the carboxylic acids $\mathbf{3 6 - 3 8}$ in a yield of ca. $80 \%$ as shown in Scheme 4 . The monophenol hydroxyl linker 5 was then functionalised with the carboxylic acid diphenylamine derivatives (36-38) to prepare a series of mixed amine-phenols (39-41) as shown in (see Series 3, Table 3). In addition, the bis(diphenylamines) (42-44) were also generated by the reaction of carboxylic acid diphenylamine derivatives with the first generation diol linker 6 .

\subsection{Oxidative stability studies}

To assess the antioxidant potential, 8, 9, 11 and $\mathbf{1 3}$ were blended into a synthetic lubricant base oil - Durasyn 164 (a polyalphaolefin, hydrogenated hydrocarbon base oil composed of dec-1-ene trimers typically used in lubricating oils). At this stage it was found that the miscibility of the bis(diphenylamines) $\mathbf{9}$ and $\mathbf{1 3}$ in the lubricant base oil was poor and hence oxidative stability analysis was not feasible. In comparison, the mixed amine-phenols exhibited better solubility even though an extended heating period was required to ensure full 
dissolution. Current commercial antioxidants Irganox L135 and Irganox L57 were used as a comparison and samples were prepared by blending of $0.5 \% \mathrm{w} / \mathrm{w}$ of each antioxidant in 50 $\mathrm{mL}$ of the lubricant base oil. The blends were analysed using (PDSC) to monitor the heat effects associated with phase transitions and chemical reactions as a function of temperature. Oxidation induction time (OIT) and oxidation onset temperature (OOT) were utilised to study the effect of antioxidants on the stability of the oil sample. OIT analysis revealed that the presence of $\mathbf{8}$ and $\mathbf{1 1}$ in the base oil had resulted in increase in the stability of the sample as shown in Figure 4. The induction time was increased from $<1$ minute for the unblended base oil to ca. 10-13 minutes for the blended samples. In addition, 8 and $\mathbf{1 1}$ showed superior performance to both commercial antioxidants, Irganox L135 and Irganox L57. However, the synergistic effect of having both functionalities on the same compound did not outweigh the effect of blending both together as evident in the L135/L57 blend (ca. 16 minutes). The methylene spacer in the mixed amine-phenol $\mathbf{1 1}$ appeared to have a positive effect on the antioxidant capabilities, revealing an induction time of ca. 3 minutes longer than 8. OOT analysis was also performed and the results for each oil blend are shown in Figure $\mathbf{5}$ where again, a significant increase in temperature $\left(\mathrm{ca} .251^{\circ} \mathrm{C}\right)$ was observed for both 8 and 11 when incorporated into the blend compared to the base oil in isolation (ca. $223^{\circ} \mathrm{C}$ ). The above results revealed that these compounds did have antioxidant capabilities but physical properties such as solubility was an issue. OIT analysis of $\mathbf{2 4 - 2 6}$ bearing an additional alkyl chain for enhanced solubility were then carried out that revealed a difference in the antioxidant capabilities of each compound (Figure 6) demonstrating that there was clearly a favoured substitution position on the ring for enhanced antioxidant properties. The mixed amine-phenol 24 (ca. 7 minutes) exhibited the quickest OIT in comparison to 25 and 26 and this poor performance can be attributed to the ortho substitution which allowed intramolecular hydrogen bonding between the aromatic amine proton and the carbonyl oxygen from the 
ester moiety. In comparison, the mixed-amine phenol 25, which was substituted in the meta position, revealed the best oxidation induction time of 13 minutes. Interestingly, this result still was not greater than a simple $1: 1$ blend $(0.25 \%$ w/w of each of Irganox L135 and Irganox L57) of Irganox L135 and Irganox L57 (ca. 16 minutes), however, some improvement was observed from Series 1. A direct comparison was made between the oxidation induction times from Series 1 and $\mathbf{2}$ to determine the structural characteristics that were contributing to an increase in induction time (Figure 7). Comparison of the meta substituted mixed aminephenols 8 and $\mathbf{2 5}$ (from Series $\mathbf{1}$ and 2, respectively) revealed that an increase in oxidation time was observed upon addition of an alkyl chain. It was proposed that this increase was associated with an improved solubility in the lubricant base oil and hence better diffusion within the medium was achieved. Additionally, comparison of the para substituted mixed amine-phenols $\mathbf{1 1}$ and $\mathbf{2 6}$ revealed that incorporation of a methylene spacer between the aromatic ring and the ester moiety had an even greater effect on the OIT than just the addition of a solubilising group. This was believed to be a result of a change in the electronics of the system and instead of the strongly electron-withdrawing ester moiety, the methyl spacer provided some electron-donating capacity to the aromatic ring. Oxidation onset temperature was also analysed and revealed the same trend of increasing oxidative stability from 24 (ortho) to 26 (para) to 25 (meta) (Figure 8). The ortho substituted mixed amine-phenol 24 revealed an oxidation onset temperature of ca. $243{ }^{\circ} \mathrm{C}$ that was significantly less than that observed for the Irganox L57 (ca. $249{ }^{\circ} \mathrm{C}$ ). The para and meta substituted mixed aminephenols (26 and 25 , respectively), however, revealed the ability to perform in the higher temperature region associated with diphenylamines. Oxidative stability analysis of the bis(diphenylamines) proved more challenging as the solubility in the lubricant base oil remained an issue even with the additional butyl chain. OIT analysis (Figure 9) revealed very poor results for the series of bis(diphenylamines) whereby only $\mathbf{2 8}$ was able to stabilise the 
base oil for more than 3 minutes. OOT analysis also revealed the same trend where 28 provided the best stabilisation out of the series, however, it was noted that none of the new species were able to perform better than Irganox L57. The poor dispersion within the lubricant oil significantly hindered the radical scavenging ability of this series of bis(diphenylamines) and it was proposed that an additional branched alkyl unit would need to be incorporated to disrupt the ordered structures and hence improve solubility. These results did, however, give confirmation that there was a synergistic effect occurring in the mixed amine-phenols where both induction time and onset temperature were significantly increased through incorporation of a phenolic moiety. To conclude the series, (meta $\left.-\mathrm{CH}_{2} \mathrm{CH}_{2}-\right)$, (para $\left.-\mathrm{CH}_{2}-\right)$ and (para $\left.\mathrm{CH}_{2} \mathrm{CH}_{2}-\right)$ substituted mixed amine-phenols with a butyl chain were synthesised and their antioxidant capabilities were investigated. OIT analysis of 39-41 revealed a dramatic increase in the stabilisation capabilities of all three compounds where the induction time for each exceeded that of the Irganox L135/57 1:1 blend as shown in Figure 10. The highest induction time of ca. 24 minutes was revealed for the mixed amine-phenol 39 possessing an ethyl spacer between the aromatic ring and the ester moiety. Interestingly, Liu et al. have also reported [36] similar OIT results for their Schiff base bridged phenolic diphenylamine antioxidants with a maximum value of ca. 25 minutes. An increase of ca. 4 minutes was observed from $\mathbf{4 0}$ (ca. 23 minutes) to $\mathbf{4 1}$ (ca. 19 minutes) where an additional methylene unit was introduced. This result indicated that stabilisation of the secondary amine, through electron donation, was a significant factor in increasing the antioxidant capabilities of these compounds. It was also proposed that the ethyl spacers provided the ideal contact distance between the diphenylamine and the phenol, therefore potentially increasing the ability of amine regeneration. A direct comparison of the meta substituted compounds (8, 25 and 39$)$ from the three series revealed the increase in induction time associated with each of the structural developments (Figure 11). This highlighted the significance of enhanced solubility 
through the inclusion of an alkyl chain which increased the induction time by ca. 3 minutes (25). In addition, inclusion of electron donating substituents as opposed to electron withdrawing substituents increased the oxidation induction time by a further ca. 10 minutes (39). OOT analysis also revealed excellent stability properties from the mixed amine-phenols where 39,40 and $\mathbf{4 1}$ all performed ( $\mathrm{ca} .254^{\circ} \mathrm{C}$ ) in the same temperature region as the $\mathrm{L} 135 / 57$ blend (Figure 12). The bis(diphenylamines) $42-44$ also revealed similar solubility issues as seen in Series 2, however, the OIT did not exceed that of the aromatic amine Irganox L57 hence reinforcing further the excellent synergistic effects of combining both diphenylamines and sterically hindered phenols within the same compound.

\section{Conclusions}

In summary, three series of mixed amine-phenols and bis(diphenylamines) were synthesised successfully, blended into the lubricant base oil and analysed for their oxidative stability properties, highlighting interesting structure-activity relationships. The mixed amine-phenols were all soluble in the lubricant base oil at $0.5 \% \mathrm{w} / \mathrm{w}$ which was further improved through the introduction of a butyl chain on the aniline ring. Even with the additional alkyl chain, all of the bis(diphenylamines) derivatives revealed poor solubility and hence oxidation induction times and oxidation onset temperatures were all lower than the current diphenylamine antioxidant Irganox L57. Series 2 of the mixed amine-phenols revealed that meta and para substitution provided the best oxidative stability properties. These properties were further enhanced in

Series 3 through the inclusion of methyl or ethyl spacers between the diphenylamine and the ester moiety from the attachment to the mono-phenol linker. It was observed that meta substitution with an ethyl spacer provided the longest oxidation induction time of ca. 23 minutes, which was ca. 8 minutes longer than the current 1:1 synergistic blend of Irganox L135 and Irganox L57. 


\section{Experimental}

4.1. General procedure for purification, characterisation, thermal and oxidative analysis

Reagents and solvents were purchased from Sigma Aldrich and used without further purification except for 3-(3,5-di-tert-butyl-4-hydroxy-phenyl)-propionic acid 7 which was purchased from Alfa Aesar. All solvents were dried and freshly distilled prior to use. Tetrahydrofuran (THF) was distilled under a nitrogen atmosphere from sodium and benzophenone. Dichloromethane was distilled under a nitrogen atmosphere from calcium hydride. Thin layer chromatography (TLC) was performed on aluminium sheets coated with Merck silica gel $60 \mathrm{~F}_{24}$. Spots were visualised under ultra-violet light $(254 \mathrm{~nm})$ with potassium permanganate as the visualising agent. Column chromatography was performed using Merck silica gel 60 (40-63 $\mu \mathrm{m}$ particle size) and a mobile phase as specified. Melting points were recorded using a Stuart MP10 melting point apparatus. ${ }^{1} \mathrm{H}$ NMR and ${ }^{13} \mathrm{C}$ NMR spectra were recorded using either $\mathrm{CDCl}_{3}$ or $\mathrm{DMSO}_{-} \mathrm{d}_{6}$ as solvent on either a Bruker Nanobay 400 or Bruker DPX 400 operating at $400 \mathrm{MHz}$ for ${ }^{1} \mathrm{H}$ NMR or at $100 \mathrm{MHz}$ for ${ }^{13} \mathrm{C}$ NMR. Infrared (IR) spectroscopic analysis was carried out using a Perkin Elmer 100 FT-IR instrument with a diamond ATR sampling attachment with samples either as solids or oils. Mass spectrometry analysis was carried out on a Thermo-Fisher Scientific Orbitrap XL LC-MS. Samples were prepared as methanol solutions $(1 \mathrm{mg} / \mathrm{mL})$ and were ionised using electrospray ionisation (ESI) and the parent mass ions were quoted. Thermogravimetric analysis (TGA) was performed using a TA instrument TGA 2950. TGA was carried out under a nitrogen atmosphere from ambient temperature to $500{ }^{\circ} \mathrm{C}$ at a rate of $10{ }^{\circ} \mathrm{C} / \mathrm{min}$ using a sample of approximately $10 \mathrm{mg}$. Pressurised differential scanning calorimetry (PDSC) was carried out at the BP Technology Centre, Pangbourne. Oxidation induction time (OIT) was performed using a TA instrument Q10 (0010-0141) or Q20 (0020P-0137). The industry standard CEC L 085-99 method was followed whereby $2 \mathrm{mg}$ of sample was added to an aluminium crucible. 
The cell was pressurised to 6.9 bar with cylinder air and the temperature was raised to $50{ }^{\circ} \mathrm{C}$ and held isothermally for 5 minutes. The temperature was then ramped at $20{ }^{\circ} \mathrm{C} / \mathrm{min}$ to 210 ${ }^{\circ} \mathrm{C}$ and held isothermally until the oxidation of the sample was induced. The time of onset of the exotherm minus the time taken to reach $210{ }^{\circ} \mathrm{C}$ was reported. PDSC oxidation onset temperature (OOT) was performed using a TA instrument 2910. An in-house method was used whereby $0.5 \mathrm{mg}$ of sample was added to an aluminium crucible. The cell was pressurised to 34.5 bar with cylinder air with a flow of $60 \mathrm{~mL} / \mathrm{min}$. The temperature was raised to $50{ }^{\circ} \mathrm{C}$ and was allowed to stabilise before heating at $50{ }^{\circ} \mathrm{C} / \mathrm{min}$ to $350{ }^{\circ} \mathrm{C}$. The temperature at which the oxidation exotherm occurred was reported. All tests were carried out in duplicate and error bars in the figures represent the standard deviations.

\subsection{Synthesis}

\subsubsection{Preparation of methyl 3-(phenylamino)benzoate (3) [40]}

In a $25 \mathrm{~mL}$ round-bottomed flask, toluene $(10 \mathrm{~mL})$ was degassed with argon for 20 minutes. Under a flow of argon, $\mathrm{Pd}(\mathrm{OAc})_{2}(0.05 \mathrm{~g}, 0.23 \mathrm{mmol})$, BINAP $(0.23 \mathrm{~g}, 0.37 \mathrm{mmol})$ and $\mathrm{Cs}_{2} \mathrm{CO}_{3}$ (2.12 mmol, $6.51 \mathrm{mmol}$ ) were added and left to stir for a further 20 minutes. To the mixture, methyl (3-bromobenzoate) 1 (1.01 g, $4.65 \mathrm{mmol})$ and aniline $2(0.51 \mathrm{~mL}, 5.58 \mathrm{mmol})$ were added. The reaction was stirred at $120^{\circ} \mathrm{C}$ and monitored by thin layer chromatography (TLC) for 24 hours. Upon completion, the reaction was cooled to room temperature and filtered through Celite ${ }^{\circledR}$, with hexane as the eluent, followed by concentration under reduced pressure. The residue was purified by flash column chromatography on silica eluting with hexane/ethyl acetate $(90: 10)\left(R_{f}=0.23\right)$ to afford $0.87 \mathrm{~g}(82 \%)$ of 3 as a pale yellow solid; M.p. $110-112{ }^{\circ} \mathrm{C}$ (literature M.p. $\left.110-11{ }^{\circ} \mathrm{C}\right)[40] ;{ }^{1} \mathrm{H}$ NMR $\left(400 \mathrm{MHz} / \mathrm{CDCl}_{3}\right) / \mathrm{ppm}, \delta=3.90$ (s, 3H,-COOCH 3 ), $5.81(\mathrm{~s}, 1 \mathrm{H},-\mathrm{NH}), 6.98$ (app. t, $1 \mathrm{H}$, app J=8 Hz, $\left.\mathrm{CH}_{\text {para }} \mathrm{C}_{4} \mathrm{H}_{4}-\mathrm{NH}\right), 7.09$ (app. d, 2H, $\left.\mathrm{CH}_{\text {para }} \mathrm{C}_{2} \mathrm{H}_{2 m e t a} \mathrm{C}_{2} \mathrm{H}_{2 o r t h o}-\mathrm{NH}\right), 7.23$ (m, 1H, HN-CH-C(COOCH 3$)-\mathrm{CHCHCH}$ ), 7.29 
(m, 3H, $\left.\mathrm{C}_{2} \mathrm{H}_{20 r t h o}-\mathrm{HN}-\mathrm{CH}-\mathrm{C}\left(\mathrm{COOCH}_{3}\right)-\mathrm{CHCHCH}-\right), 7.57$ (app. d, $1 \mathrm{H}, \mathrm{HN}-\mathrm{CH}-\mathrm{C}\left(\mathrm{COOCH}_{3}\right)-$ $\mathrm{CH}), 7.72\left(\mathrm{~m}, 1 \mathrm{H}, \mathrm{HN}-\mathrm{CH}-\mathrm{C}\left(\mathrm{COOCH}_{3}\right)\right) ;{ }^{13} \mathrm{C} \mathrm{NMR}\left(100 \mathrm{MHz} / \mathrm{CDCl}_{3}\right) / \mathrm{ppm}, \delta=52.2,118.2$, $118.4,121.5,121.77,121.80,129.4,129.3,131.4,142.4,143.6,167.1$; Found $[\mathrm{M}+\mathrm{H}]^{+}$ $\left(\mathrm{C}_{14} \mathrm{H}_{14} \mathrm{NO}_{2}\right) \mathrm{m} / \mathrm{z}=228.1019$ (Calc. 228.1025); IR (ATR) $v / \mathrm{cm}^{-1}: 3358,3030,1694,1578$, $1294,744$.

\subsubsection{Preparation of 3-(phenylamino)benzoic acid (4) [41]}

To a solution of $3(0.50 \mathrm{~g}, 2.20 \mathrm{mmol})$ in ethanol $(10 \mathrm{~mL})$, aqueous potassium hydroxide $(0.25$ $\mathrm{g}, 4.40 \mathrm{mmol}$ ) was added at room temperature. The mixture was then allowed to stir at 100 ${ }^{\circ} \mathrm{C}$ for a minimum of 1 hour and was monitored closely by TLC until consumption of $\mathbf{3}$ was observed. Upon completion, the reaction was allowed to cool and the ethanol was removed in vacuo. The remaining solution was cooled to $0^{\circ} \mathrm{C}$ and acidified to $\mathrm{pH} 2$ with $2 \mathrm{M} \mathrm{HCl}$. The resulting precipitate was collected via vacuum filtration and washed with water $(15 \mathrm{ml})$. The precipitate was then dissolved in chloroform $(20 \mathrm{~mL})$ and washed with brine $(20 \mathrm{~mL})$. The organic layer was dried over $\mathrm{MgSO}_{4}$, filtered and the solvent removed in vacuo to yield 0.38 g (81\%) of 4 as a white solid; M.p. $140-142{ }^{\circ} \mathrm{C}$ (literature M.p. $140-141{ }^{\circ} \mathrm{C}$ ) [41]; ${ }^{1} \mathrm{H}$ NMR (400 $\left.\mathrm{MHz} / \mathrm{CDCl}_{3}\right) / \mathrm{ppm}, \delta=6.99$ (app. t, $1 \mathrm{H}$, app $J=8 \mathrm{~Hz}, \mathrm{CH}_{\text {para }} \mathrm{C}_{4} \mathrm{H}_{4}-\mathrm{NH}$ ), 7.10 (app. d, 2H, $\left.\mathrm{CH}_{\text {para }} \mathrm{C}_{2} \mathrm{H}_{2 m e t a} \mathrm{C}_{2} \mathrm{H}_{2 o r t h o}-\mathrm{NH}\right), 7.30$ (m, 4H, $\mathrm{C}_{2} \mathrm{H}_{2 o r t h o}-\mathrm{HN}-\mathrm{CH}-\mathrm{C}(\mathrm{COOH})-\mathrm{CHCHCH}$ ), 7.64 (app. d, $\quad 1 \mathrm{H}, \quad \mathrm{HN}-\mathrm{CH}-\mathrm{C}(\mathrm{COOH})-\mathrm{CH}), 7.77\left(\mathrm{~m}, \quad 1 \mathrm{H}, \quad \mathrm{HN}-\mathrm{CH}-\mathrm{C}\left(\mathrm{COOCH}_{3}\right)\right) ;{ }^{13} \mathrm{C} \mathrm{NMR} \quad(100$ $\left.\mathrm{MHz} / \mathrm{CDCl}_{3}\right) / \mathrm{ppm}, \delta=118.5,118.6,122.0,122.2,129.5,129.6,130.5,142.2,143.8,172.2$; Found $[\mathrm{M}+\mathrm{H}]^{+}\left(\mathrm{C}_{13} \mathrm{H}_{12} \mathrm{NO}_{2}\right) \mathrm{m} / \mathrm{z}=214.0863$ (Calc. 214.0868); IR (ATR) $v / \mathrm{cm}^{-1}: 3398,2992-$ 2540 (br), 1682, 1584, 1464, 1294, 748.

\subsubsection{Preparation of the mono-phenolic linker (5)}

The first generation hydroxyl linker $6(5.01 \mathrm{~g}, 22.3 \mathrm{mmol}), N_{,} N^{\prime}$-dicyclohexylcarbodiimide (DCC) $(0.92 \mathrm{~g}, 4.46 \mathrm{mmol})$ and DPTS (60\%) were dissolved in dry dichloromethane $(50 \mathrm{~mL})$ 
and cooled to $0{ }^{\circ} \mathrm{C}$. To this, a solution of 3-(3,5-di-tert-butyl-4-hydroxy-phenyl)-propionic acid $7(1.24 \mathrm{~g}, 4.46 \mathrm{mmol})$ in dry dichloromethane $(20 \mathrm{~mL})$ was added dropwise to the solution. The reaction was left to stir at room temperature and was monitored closely by TLC (90:10 hexane/ethyl acetate) until any small formation of the diphenol was observed $(R f=0.38)$. The reaction mixture was filtered to remove the white $N, N^{\prime}$-dicyclohexylurea (DCU) precipitate and the filtrate was concentrated in vacuo. The crude product was immediately subjected to flash column chromatography on silica eluting with hexane/ethyl acetate $(80: 20)\left(R_{f}=0.24\right)$ to afford $2.15 \mathrm{~g}(95 \%)$ of 5 as a colourless oil; ${ }^{1} \mathrm{H}$ NMR $\left(400 \mathrm{MHz} / \mathrm{CDCl}_{3}\right) / \mathrm{ppm}, \delta=0.89$ (t, $\left.6 \mathrm{H}, \quad-\mathrm{CH}_{2} \mathrm{CH}_{3}\right), \quad 1.15\left(\mathrm{~s}, \quad 3 \mathrm{H}, \quad-\mathrm{CH}_{3}\right), \quad 1.28-1.37 \quad\left(\mathrm{~m}, \quad 8 \mathrm{H}, \quad \mathrm{COO}-\mathrm{CH}_{2}-\mathrm{CH}\left(\mathrm{CH}_{2} \mathrm{CH}_{3}\right)-\right.$ $\left.\mathrm{CH}_{2} \mathrm{CH}_{2} \mathrm{CH}_{2} \mathrm{CH}_{3}\right), 1.43$ (s, $18 \mathrm{H}, \mathrm{CH}_{3}$ tert-butyl), 1.59 (m, $\left.1 \mathrm{H}, \mathrm{COO}-\mathrm{CH}_{2}-\mathrm{CH}\left(\mathrm{CH}_{2} \mathrm{CH}_{3}\right)-\mathrm{C}_{4} \mathrm{H}_{9}\right)$, 2.53 (s(br), 1H, -OH), 2.62 (t, 2H, Ar- $\left.\mathrm{CH}_{2} \mathrm{CH}_{2}-\mathrm{COO}-\right), 2.86$ (t, 2H, $\left.\mathrm{Ar}-\mathrm{CH}_{2} \mathrm{CH}_{2}-\mathrm{COO}-\right), 3.57$ (m, $2 \mathrm{H}, \mathrm{HO}-\mathrm{CH}_{2}-\mathrm{C}$ ) $), 4.05\left(\mathrm{~m}, 2 \mathrm{H}, \mathrm{COO}-\mathrm{CH}_{2}-\mathrm{CH}\left(\mathrm{CH}_{2} \mathrm{CH}_{3}\right)-\mathrm{C}_{4} \mathrm{H}_{9}\right), 4.20$ (d, $1 \mathrm{H}, \mathrm{COO}-\mathrm{CH}-\mathrm{C}$ equatorial), 4.33 (d, 1H, COO-CH-C axial), 5.09 (s, 1H, Ar- $\mathrm{OH}), 6.98(\mathrm{~s}, 2 \mathrm{H}, \mathrm{Ar}-\mathrm{CH}) ;{ }^{13} \mathrm{C} \mathrm{NMR}$ $\left(100 \mathrm{MHz} / \mathrm{CDCl}_{3}\right) / \mathrm{ppm}, \delta=11.0,14.1,17.6,23.0,23.8,28.9,30.4,30.9,34.3,36.3,38.7$, 48.3, 64.9, 65.7, 67.3, 124.7, $130.7,136.0,152.3,173.4,174.6$; Found $[\mathrm{M}+\mathrm{Na}]^{+}\left(\mathrm{C}_{30} \mathrm{H}_{50} \mathrm{O} 6 \mathrm{Na}\right)$ $\mathrm{m} / \mathrm{z}=529.3500$ (Calc. 529.3505); IR (ATR) $v / \mathrm{cm}^{-1}:$ 3528, 2960, 1726, 1140, 1042.

\subsubsection{Preparation of the mixed amine-phenol (8)}

The mono-phenolic linker 5 (0.34 g, $0.67 \mathrm{mmol}$ ), 3-(phenylamino)benzoic acid 4 (0.16 g, 0.74 $\mathrm{mmol})$ and DPTS $(60 \%)$ were dissolved in dry dichloromethane $(10 \mathrm{~mL})$ and stirred at room temperature for 30 minutes. To the solution, DCC $(0.15 \mathrm{~g}, 0.74 \mathrm{mmol})$ dissolved in dry dichloromethane $(10 \mathrm{~mL})$ was added over 15 minutes. The reaction was left overnight at room temperature under a nitrogen atmosphere. The reaction mixture was filtered to remove the white DCU precipitate and the filtrate was concentrated. The residue was purified by flash column chromatography on silica eluting with hexane/ethyl acetate $(80: 20)\left(R_{f}=0.32\right)$ to afford $0.31 \mathrm{~g}(46 \%)$ of 8 as a viscous yellow oil; ${ }^{1} \mathrm{H} \mathrm{NMR}\left(400 \mathrm{MHz} / \mathrm{CDCl}_{3}\right) / \mathrm{ppm}, \delta=0.84(\mathrm{t}$, 
$\left.6 \mathrm{H},-\mathrm{CH}_{2} \mathrm{CH}_{3}\right), 1.23-1.36\left(\mathrm{~m}, 12 \mathrm{H}\right.$, alkyl $\left.-\mathrm{CH}_{2},-\mathrm{CH}_{3}\right), 1.42\left(\mathrm{~s}, 18 \mathrm{H}, \mathrm{CH}_{3}\right.$ tert-butyl), 1.56 (m, $1 \mathrm{H},-\mathrm{CH}$ ), 2.60 (t, 2H, Ar- $\mathrm{CH}_{2} \mathrm{CH}_{2}-\mathrm{COO}$ ), 2.85 (t, 2H, Ar- $\mathrm{CH}_{2} \mathrm{CH}_{2}-\mathrm{COO}$ ), 4.04 (m, 2H, COO$\left.\mathrm{CH}_{2}-\mathrm{CH}\left(\mathrm{CH}_{2} \mathrm{CH}_{3}\right)-\mathrm{C}_{4} \mathrm{H}_{9}\right), 4.34$ (s, $\left.2 \mathrm{H}, \mathrm{Ar}-\mathrm{COO}-\mathrm{CH}_{2}-\mathrm{C}\right), 4.44$ (s, 2H, Ar-COO-CH$\left.{ }_{2}-\mathrm{C}\right), 5.08$ (s, $1 \mathrm{H},-\mathrm{OH}), 5.87(\mathrm{~s}, 1 \mathrm{H},-\mathrm{NH}), 6.97\left(\mathrm{~m}, 3 \mathrm{H}, \mathrm{ArCH}_{\text {para }} \mathrm{C}_{4} \mathrm{H}_{4}-\mathrm{NH}\right.$ and $\left.\mathrm{Ph}-\mathrm{CH}\right), 7.09$ (app. d, 2H, $\left.\mathrm{ArCH}_{\text {para }} \mathrm{C}_{2} \mathrm{H}_{2 m e t a} \mathrm{C}_{2} \mathrm{H}_{2 o r t h o}-\mathrm{NH}\right), 7.30$ (m, 4H, $\left.\mathrm{ArC}_{2} \mathrm{H}_{2 o r t h o}-\mathrm{HN}-\mathrm{ArCH}-\mathrm{C}(\mathrm{COOH})-\mathrm{CHCHCH}-\right)$, 7.50 (app. d, 1H, HN-ArCH-C(COOH)-CH), 7.67 (m, 1H, HN-ArCH-C(COOCH 3$) ;{ }^{13} \mathrm{C}$ NMR $\left(100 \mathrm{MHz} / \mathrm{CDCl}_{3}\right) / \mathrm{ppm}, \delta=11.0,14.1,18.0,22.9,23.7,28.9,30.3,30.9,34.3,36.2,38.7$, $46.6,65.6,66.1,67.5,118.0,118.5,121.5,121.7,121.9,124.7,129.4,129.5,130.8,136.0$, 142.2, 143.7, 152.2, 166.0, 172.7, 172.9; Found $[\mathrm{M}+\mathrm{Na}]^{+}\left(\mathrm{C}_{43} \mathrm{H}_{59} \mathrm{NO}_{7} \mathrm{Na}\right) \mathrm{m} / \mathrm{z}=724.4184$ (Calc. 724.4189); IR (ATR) v/cm-1: 3641, 3385, 2960, 1731, 1517, 1132, 749.

\subsubsection{Preparation of the bis(diphenylamine) (9)}

The first generation hydroxyl linker $6(0.28 \mathrm{~g}, 1.12 \mathrm{mmol})$, 3-(phenylamino)benzoic acid 4 $(0.50 \mathrm{~g}, 2.34 \mathrm{mmol})$ and DPTS $(60 \%)$ were dissolved in dry dichloromethane $(20 \mathrm{~mL})$. To the solution, DCC $(0.48 \mathrm{~g}, 2.34 \mathrm{mmol})$ dissolved in dry dichloromethane $(10 \mathrm{~mL})$ was added over 15 minutes. The reaction was left overnight at room temperature under a nitrogen atmosphere. The reaction mixture was filtered to remove the white DCU precipitate and the filtrate was concentrated. The crude product was purified by flash column chromatography on silica eluting with hexane/ethyl acetate $(80: 20)\left(R_{f}=0.4\right)$ to afford $0.47 \mathrm{~g}(66 \%)$ of 9 as a viscous orange oil; ${ }^{1} \mathrm{H} \mathrm{NMR}\left(400 \mathrm{MHz} / \mathrm{CDCl}_{3}\right) / \mathrm{ppm}, \delta=0.81\left(\mathrm{~m}, 6 \mathrm{H},-\mathrm{CH}_{2} \mathrm{CH}_{3}\right), 1.20-1.33(\mathrm{~m}$, 8H, alkyl - $\left.\mathrm{CH}_{2}\right), 1.41\left(\mathrm{~s}, 3 \mathrm{H},-\mathrm{CH}_{3}\right), 1.54(\mathrm{~m}, 1 \mathrm{H},-\mathrm{CH}), 4.06\left(\mathrm{~m}, 2 \mathrm{H}, \mathrm{COO}-\mathrm{CH}_{2}-\mathrm{CH}\left(\mathrm{CH}_{2} \mathrm{CH}_{3}\right)-\right.$ $\left.\mathrm{C}_{4} \mathrm{H}_{9}\right), 4.55$ (s, 4H, Ar-COO- $\left.\mathrm{CH}_{2}-\mathrm{C}\right), 5.79$ (s(br), 2H, $\left.-\mathrm{NH}\right), 6.97$ (app. t, 2H, app. J = $8 \mathrm{~Hz}$, $\mathrm{CH}_{\text {para }} \mathrm{C}_{4} \mathrm{H}_{4}-\mathrm{NH}$ ), 7.08 (app. d, 4H, app. $\left.\mathrm{J}=8 \mathrm{~Hz}, \mathrm{CH}_{\text {para }} \mathrm{C}_{2} \mathrm{H}_{2 m e t a} \mathrm{C}_{2} \mathrm{H}_{2 o r t h o}-\mathrm{NH}\right), 7.29(\mathrm{~m}, 8 \mathrm{H}$, $\left.\mathrm{C}_{2} \mathrm{H}_{2 o r t h o}-\mathrm{HN}-\mathrm{CH}-\mathrm{C}\left(\mathrm{COOCH}_{3}\right)-\mathrm{CHCHCH}-\right), 7.51$ (app. d, $2 \mathrm{H}$, app. $J=4 \mathrm{~Hz}, \mathrm{HN}-\mathrm{CH}-$

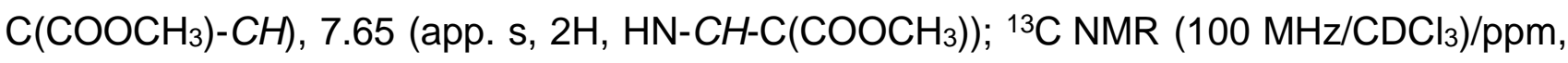
$\delta=10.9,14.0,18.1,22.9,23.7,28.9,30.3,38.7,46.8,66.3,67.5,118.1,118.5,121.4,121.7$, 
$121.9,129.5,130.8,142.2,146.7,166.0,172.9 ;$ Found $[\mathrm{M}+\mathrm{H}]^{+}\left(\mathrm{C}_{39} \mathrm{H}_{45} \mathrm{~N}_{2} \mathrm{O}_{6}\right) \mathrm{m} / \mathrm{z}=637.3272$ (Calc. 637.3278); IR (ATR) v/cm $1: 3380,2960,1714,1590,1494,1204,1104,746$.

\subsubsection{Preparation of mixed amine-phenol (11)}

The mono-phenolic linker 5 (0.74 g, $1.46 \mathrm{mmol})$, 2-(4-(phenylamino)phenyl)acetic acid 12 $(0.50 \mathrm{~g}, 2.20 \mathrm{mmol})$, DPTS $(60 \%)$ and DCC $(0.45 \mathrm{~g}, 2.20 \mathrm{mmol})$ were allowed to react according to the general mixed amine-phenol procedure. The crude product was purified by flash column chromatography on silica eluting with hexane/ethyl acetate $(80: 20)\left(R_{f}=0.31\right)$ to afford $0.75 \mathrm{~g}(72 \%)$ of 11 as a viscous pale yellow oil; ${ }^{1} \mathrm{H} \mathrm{NMR}\left(400 \mathrm{MHz} / \mathrm{CDCl}_{3}\right) / \mathrm{ppm}, \delta=$ $0.87\left(\mathrm{~m}, 6 \mathrm{H},-\mathrm{CH}_{2} \mathrm{CH}_{3}\right), 1.16\left(\mathrm{~s}, 3 \mathrm{H},-\mathrm{CH}_{3}\right), 1.31-1.36\left(\mathrm{~m}, 8 \mathrm{H}\right.$, alkyl $\left.-\mathrm{CH}_{2}\right), 1.42\left(\mathrm{~s}, 18 \mathrm{H}, \mathrm{CH}_{3}\right.$ tert-butyl), 1.54 (m, 1H, -CH), 2.57 (t, 2H, J = $\left.8 \mathrm{~Hz}, \mathrm{Ar}_{-} \mathrm{CH}_{2} \mathrm{CH}_{2} \mathrm{COO}\right), 2.83$ (t, $2 \mathrm{H}, J=8 \mathrm{~Hz}$, Ar- $\mathrm{CH}_{2} \mathrm{CH}_{2}-\mathrm{COO}$ ), 3.54 (s, $\left.2 \mathrm{H}, \mathrm{Ar}-\mathrm{CH}_{2}-\mathrm{COO}\right), 4.01\left(\mathrm{~m}, 2 \mathrm{H}, \mathrm{COO}-\mathrm{CH}_{2}-\mathrm{CH}\left(\mathrm{CH}_{2} \mathrm{CH}_{3}\right)-\mathrm{C}_{4} \mathrm{H}_{9}\right)$, $4.22\left(\mathrm{~m}, 4 \mathrm{H}, \mathrm{CH}_{2}-\mathrm{COO}-\mathrm{CH}_{2}-\mathrm{C}\right), 5.07$ (s, 1H, $\mathrm{ArOH}$ ), 5.68 (s, 1H, -NH), 6.92 (app. t, 1H, app. $\left.J=8 \mathrm{~Hz}, \mathrm{CH}_{\text {para }} \mathrm{C}_{4} \mathrm{H}_{4}-\mathrm{NH}\right), 6.97(\mathrm{~s}, 2 \mathrm{H}, \mathrm{Ph}-\mathrm{CH}), 7.03\left(\mathrm{~m}, 4 \mathrm{H}, \mathrm{ArCH} \mathrm{H}_{\text {ortho }}-\mathrm{NH}-\mathrm{ArCH}\right.$ ortho), 7.14 (app. d, 2H, J = $\left.12 \mathrm{~Hz}, \mathrm{NH}-\mathrm{ArCHCH}-\mathrm{C}\left(\mathrm{CH}_{2} \mathrm{COO}-\right)\right), 7.25$ (app. t, 4H, J $=8 \mathrm{~Hz}$, $\left.\mathrm{ArCH}_{\text {para }} \mathrm{CH}_{\text {meta }} \mathrm{CH}_{\text {ortho }} \mathrm{NH}\right) ;{ }^{13} \mathrm{C} \mathrm{NMR}\left(100 \mathrm{MHz} / \mathrm{CDCl}_{3}\right) / \mathrm{ppm}, \delta=10.9,14.0,17.8,22.9,23.7$, $28.9,30.3,30.8,34.3,36.2,38.7,40.5,46.4,65.4,65.8,67.4,117.8,121.0,124.7,126.0$, $129.3,130.2,130.8,136.0,142.2,143.0,152.2,171.3,172.6,172.8$; Found $[\mathrm{M}+\mathrm{H}]^{+}$ $\left(\mathrm{C}_{44} \mathrm{H}_{62} \mathrm{NO}_{7}\right) \mathrm{m} / \mathrm{z}=716.4524$ (Calc. 716.4526$) ;$ IR (ATR) $v / \mathrm{cm}^{-1}: 3641,3385,2960,1731$, 1517, 1132, 749.

\subsubsection{Preparation of bis(diphenylamine) (13)}

The first generation hydroxyl linker 6 (0.43 g, $1.91 \mathrm{mmol}), 2-(4-($ phenylamino)phenyl)acetic acid 12 (1.01 g, $4.40 \mathrm{mmol})$, DPTS (60\%) and DCC (0.91 g, $4.40 \mathrm{mmol})$ were allowed to react according to the general bis(diphenylamine) procedure. The crude product was purified by flash column chromatography on silica eluting with hexane/ethyl acetate (80:20) 
$\left(R_{f}=0.43\right)$ to afford $0.98 \mathrm{~g}(77 \%)$ of 13 as a viscous dark red oil; ${ }^{1} \mathrm{H}$ NMR (400 $\left.\mathrm{MHz} / \mathrm{CDCl}_{3}\right) / \mathrm{ppm}, \delta=0.86\left(\mathrm{~m}, 6 \mathrm{H},-\mathrm{CH}_{2} \mathrm{CH}_{3}\right), 1.16\left(\mathrm{~s}, 3 \mathrm{H},-\mathrm{CH}_{3}\right), 1.25-1.35(\mathrm{~m}, 8 \mathrm{H}$, alkyl $\left.\mathrm{CH}_{2}\right), 1.54(\mathrm{~m}, 1 \mathrm{H},-\mathrm{CH}), 3.52\left(\mathrm{~s}, 4 \mathrm{H}, \mathrm{Ar}-\mathrm{CH}_{2}-\mathrm{COO}\right), 3.98\left(\mathrm{~m}, 2 \mathrm{H}, \mathrm{COO}-\mathrm{CH}_{2}-\mathrm{CH}\left(\mathrm{CH}_{2} \mathrm{CH}_{3}\right)-\right.$ $\left.\mathrm{C}_{4} \mathrm{H}_{9}\right), 4.17\left(\mathrm{~d}, 2 \mathrm{H}, \mathrm{J}=8 \mathrm{~Hz}, \mathrm{Ar}-\mathrm{COO}-\mathrm{CH}_{2}-\mathrm{C}\right), 4.24$ (d, 2H, J=12 Hz, Ar-COO-CH$\left.-\mathrm{C}\right), 5.67$ (s, 2H, -NH), 6.92 (app. t, 2H, app. J = $\left.8 \mathrm{~Hz}, \mathrm{CH}_{\text {para }} \mathrm{C}_{4} \mathrm{H}_{4}-\mathrm{NH}\right), 6.99$ (app. d, 4H, app. J = 8 $\left.\mathrm{Hz}, \mathrm{NH}-\mathrm{CHCH}-\left(\mathrm{CH}_{2} \mathrm{COO}-\right)\right), 7.04$ (app. d, 4H, app. J = $\left.8 \mathrm{~Hz}, \mathrm{ArCH}_{\text {para }} \mathrm{CH}_{\text {meta }} \mathrm{CH}_{\text {ortho- }} \mathrm{NH}\right), 7.10$ (app. d, 4H, app. $\left.J=8 \mathrm{~Hz}, \mathrm{NH}-\mathrm{CHCH}-\left(\mathrm{CH}_{2} \mathrm{COO}-\right)\right), 7.25$ (app. t, 4H, app. $J=8 \mathrm{~Hz}$, $\left.\mathrm{ArCH}_{\text {para }} \mathrm{CH}_{\text {meta }} \mathrm{CH}_{\text {ortho }}-\mathrm{NH}\right) ;{ }^{13} \mathrm{C} \mathrm{NMR}\left(100 \mathrm{MHz} / \mathrm{CDCl}_{3}\right) / \mathrm{ppm}, \delta=10.9,14.1,17.9,22.9,23.7$, $28.8,30.3,38.6,40.4,46.3,65.6,67.4,117.9,121.0,126.0,129.3,130.2,142.2,143.0$, 171.3, 172.8; Found $[\mathrm{M}+\mathrm{H}]^{+}\left(\mathrm{C}_{41} \mathrm{H}_{49} \mathrm{~N}_{2} \mathrm{O}_{6}\right) \mathrm{m} / \mathrm{z}=665.3585$ (Calc. 665.3591); IR (ATR) $v / \mathrm{cm}^{-1}$ : $3385,2960,1727,1596,1516,1132,746$.

\subsubsection{Preparation of methyl 2-((4-butylphenyl)amino)benzoate (14)}

Methyl (2-bromobenzoate) 15 (1.00 g, $4.65 \mathrm{mmol})$ and 4-butylaniline 16 (0.88 mL, $5.58 \mathrm{mmol})$ were added to $\mathrm{Pd}(\mathrm{OAc})_{2}(0.02 \mathrm{~g}, 0.09 \mathrm{mmol})$, BINAP $(0.12 \mathrm{~g}, 0.18 \mathrm{mmol})$ and $\mathrm{K}_{2} \mathrm{CO}_{3}(0.90 \mathrm{~g}$, $6.51 \mathrm{mmol}$ ) and were allowed to react according to the general Buchwald-Hartwig amination procedure. The crude residue was purified by flash column chromatography on silica eluting with hexane/ethyl acetate $(80: 20)\left(R_{f}=0.38\right)$ to afford $0.70 \mathrm{~g}(53 \%)$ of 14 as a white solid; M.p. $135-137{ }^{\circ} \mathrm{C} ;{ }^{1} \mathrm{H}$ NMR $\left(400 \mathrm{MHz} / \mathrm{CDCl}_{3}\right) / \mathrm{ppm}, \delta=0.95(\mathrm{t}, 3 \mathrm{H}, J=8 \mathrm{~Hz}$, $\mathrm{CH}_{3} \mathrm{CH}_{2} \mathrm{CH}_{2} \mathrm{CH}_{2} \mathrm{Ar}$ ), 1.39 (sex., $2 \mathrm{H}, J=8 \mathrm{~Hz},-\mathrm{CH}_{3} \mathrm{CH}_{2} \mathrm{CH}_{2} \mathrm{CH}_{2} \mathrm{Ar}$ ), 1.61 (quin., $2 \mathrm{H}, \mathrm{J}=8 \mathrm{~Hz}$, $-\mathrm{CH}_{3} \mathrm{CH}_{2} \mathrm{CH}_{2} \mathrm{CH}_{2} \mathrm{Ar}$ ), 2.61 (t, $2 \mathrm{H}, \mathrm{J}=8 \mathrm{~Hz},-\mathrm{CH}_{3} \mathrm{CH}_{2} \mathrm{CH}_{2} \mathrm{CH}_{2} \mathrm{Ar}$ ), 3.91 (s, 3H,-COOCH 3 ), 6.70 (app. t, 1H, app $J=8 \mathrm{~Hz}, \mathrm{H}_{3} \mathrm{COOC}-\mathrm{CHCHCHCHC-NH}$ ), 7.17 (app. s, 4H, $\mathrm{C}_{4} \mathrm{H}_{9}-\mathrm{ArCH}$ ), 7.20 (app. d, 1H, $\mathrm{H}_{3} \mathrm{COOC}-\mathrm{CHCHCHCHC}-\mathrm{NH}$ ), 7.29 (app. t, 1H, $\mathrm{H}_{3} \mathrm{COOC}-\mathrm{CHCHCHCHC}-\mathrm{NH}$ ), 7.96 (app. d, $\left.1 \mathrm{H}, \quad \mathrm{H}_{3} \mathrm{COOC}-\mathrm{CHCHCHCHC}-\mathrm{NH}\right), \quad 9.40 \quad(\mathrm{~s}, \quad 1 \mathrm{H},-\mathrm{NH}) ; \quad{ }^{13} \mathrm{C}$ NMR $\quad(100$ $\left.\mathrm{MHz} / \mathrm{CDCl}_{3}\right) / \mathrm{ppm}, \delta=14.0,22.4,33.8,35.1,51.7,111.4,113.8,116.6,122.6,123.1,126.2$, 
129.3, 131.6, 134.1, 138.2, 138.6, 148.6, 169.0; Found $[\mathrm{M}+\mathrm{H}]^{+}\left(\mathrm{C}_{18} \mathrm{H}_{22} \mathrm{NO}_{2}\right) \mathrm{m} / \mathrm{z}=284.1645$ (Calc. 284.1651); IR (ATR) v/cm-1: 3321, 2928, 1684, 1514, 1230, 1082, 746.

\subsubsection{Preparation of methyl 3-((4-butylphenyl)amino)benzoate (17) [42]}

Methyl (3-bromobenzoate) 18 (0.50 g, $2.33 \mathrm{mmol})$ and 4-butylaniline 16 (0.44 mL, $2.79 \mathrm{mmol})$ were added to $\mathrm{Pd}(\mathrm{OAc})_{2}(0.02 \mathrm{~g}, 0.08 \mathrm{mmol})$, $\operatorname{BINAP}(0.10 \mathrm{~g}, 0.16 \mathrm{mmol})$ and $\mathrm{Cs}_{2} \mathrm{CO}_{3}(1.06 \mathrm{~g}$, $3.26 \mathrm{mmol}$ ) and were allowed to react according to the general Buchwald-Hartwig amination procedure. The crude residue was purified by flash column chromatography on silica eluting with hexane/ethyl acetate $(80: 20)\left(R_{f}=0.36\right)$ to afford $0.35 \mathrm{~g}(55 \%)$ of 17 as a pale yellow solid; M.p. $101-103{ }^{\circ} \mathrm{C}$ (literature M.p. $\left.101-103{ }^{\circ} \mathrm{C}\right)[42] ;{ }^{1} \mathrm{H} \mathrm{NMR}\left(400 \mathrm{MHz} / \mathrm{CDCl}_{3}\right) / \mathrm{ppm}, \delta=$ 0.93 (t, $3 \mathrm{H}, J=8 \mathrm{~Hz},-\mathrm{CH}_{3} \mathrm{CH}_{2} \mathrm{CH}_{2} \mathrm{CH}_{2} \mathrm{Ar}$ ), 1.36 (sex., $2 \mathrm{H}, J=8 \mathrm{~Hz},-\mathrm{CH}_{3} \mathrm{CH}_{2} \mathrm{CH}_{2} \mathrm{CH}_{2} \mathrm{Ar}$ ), 1.59 (quin., $2 \mathrm{H}, J=8 \mathrm{~Hz},-\mathrm{CH}_{3} \mathrm{CH}_{2} \mathrm{CH}_{2} \mathrm{CH}_{2} \mathrm{Ar}$ ), 2.57 (t, $2 \mathrm{H}, J=8 \mathrm{~Hz},-\mathrm{CH}_{3} \mathrm{CH}_{2} \mathrm{CH}_{2} \mathrm{CH}_{2} \mathrm{Ar}$ ), 3.89 (s, 3H,-COOCH 3 ), $5.72(\mathrm{~s}, 1 \mathrm{H},-\mathrm{NH}), 7.03$ (app. d, 2H, app. J = 8 Hz, HN-ArCHCH), 7.11 (app. d, 2H, app. $\left.\mathrm{J}=8 \mathrm{~Hz}, \mathrm{C}_{4} \mathrm{H}_{9}-\mathrm{ArCHCH}\right), 7.19$ (app. d, $1 \mathrm{H}$, app. $\mathrm{J}=8 \mathrm{~Hz}, \mathrm{HN}-\mathrm{CH}-$ $\left.\mathrm{C}\left(\mathrm{COOCH}_{3}\right)-\mathrm{CHCHCH}-\mathrm{NH}\right), 7.28$ (app. t, $\left.1 \mathrm{H}, J=8 \mathrm{~Hz}, \mathrm{HN}-\mathrm{CH}-\mathrm{C}\left(\mathrm{COOCH}_{3}\right)-\mathrm{CHCHCH}-\mathrm{NH}\right)$, 7.52 (app. d, $1 \mathrm{H}, \mathrm{J}=8 \mathrm{~Hz}, \mathrm{HN}-\mathrm{CH}-\mathrm{C}\left(\mathrm{COOCH}_{3}\right)-\mathrm{CHCHCH}-\mathrm{NH}$ ), 7.66 (app. s, $1 \mathrm{H}, \mathrm{HN}-\mathrm{CH}$ $\left.\mathrm{C}\left(\mathrm{COOCH}_{3}\right)\right) ;{ }^{13} \mathrm{C} \mathrm{NMR}\left(100 \mathrm{MHz} / \mathrm{CDCl}_{3}\right) / \mathrm{ppm}, \delta=14.0,22.4,33.8,35.0,52.1,117.4,119.3$, $120.7,121.1,129.3,129.4,131.3,136.9,139.7,144.3,167.2$; Found $[\mathrm{M}+\mathrm{H}]^{+}\left(\mathrm{C}_{18} \mathrm{H}_{22} \mathrm{NO}_{2}\right)$ $\mathrm{m} / \mathrm{z}=284.1646$ (Calc. 284.1651); IR (ATR) $v / \mathrm{cm}^{-1}: 3364,2927,1700,1288,1217,750$.

\subsubsection{Preparation of methyl 4-((4-butylphenyl)amino)benzoate (19)}

Methyl (4-iodobenzoate) 20 (1.00 g, $3.82 \mathrm{mmol})$ and 4-butylaniline $16(0.72 \mathrm{~mL}, 4.58 \mathrm{mmol})$ were added to $\mathrm{Pd}(\mathrm{OAc}) 2(0.02 \mathrm{~g}, 0.08 \mathrm{mmol})$, $\operatorname{BINAP}(0.10 \mathrm{~g}, 0.16 \mathrm{mmol})$ and $\mathrm{Cs}_{2} \mathrm{CO}_{3}(1.74$ $\mathrm{g}, 5.35 \mathrm{mmol}$ ) and were allowed to react according to the general Buchwald-Hartwig amination procedure. The crude residue was purified by flash column chromatography on silica eluting with hexane/ethyl acetate $(80: 20)\left(R_{f}=0.36\right)$ to afford $0.54 \mathrm{~g}(50 \%)$ of 19 as a 
waxy brown solid; M.p. $51-53{ }^{\circ} \mathrm{C} ;{ }^{1} \mathrm{H}$ NMR $\left(400 \mathrm{MHz} / \mathrm{CDCl}_{3}\right) / \mathrm{ppm}, \delta=0.94$ (t, 3H, J = $8 \mathrm{~Hz}$, $\mathrm{CH}_{3} \mathrm{CH}_{2} \mathrm{CH}_{2} \mathrm{CH}_{2} \mathrm{Ar}$ ), 1.38 (sex., $2 \mathrm{H}, \mathrm{J}=8 \mathrm{~Hz},-\mathrm{CH}_{3} \mathrm{CH}_{2} \mathrm{CH}_{2} \mathrm{CH}_{2} \mathrm{Ar}$ ), 1.61 (quin., $2 \mathrm{H}, \mathrm{J}=8 \mathrm{~Hz}$, $-\mathrm{CH}_{3} \mathrm{CH}_{2} \mathrm{CH}_{2} \mathrm{CH}_{2} \mathrm{Ar}$ ), 2.59 (t, $2 \mathrm{H}, J=8 \mathrm{~Hz},-\mathrm{CH}_{3} \mathrm{CH}_{2} \mathrm{CH}_{2} \mathrm{CH}_{2} \mathrm{Ar}$ ), 3.87 (s, 3H,-COOCH $), 5.94$ (s, 1H, -NH), 6.93 (app. d, 2H, app. J=12 Hz, HN-ArCHCH-CHCH-C(COOCH 3$)$ ), 7.09 (app. d, $2 \mathrm{H}$, app. $\mathrm{J}=8 \mathrm{~Hz}, \mathrm{HN}-\mathrm{Ar} C H C H), 7.15$ (app. d, $1 \mathrm{H}$, app. $\left.\mathrm{J}=8 \mathrm{~Hz}, \mathrm{C}_{4} \mathrm{H}_{9}-\mathrm{ArCHCH}\right), 7.89$ (app. d, $\left.2 \mathrm{H}, J=8 \mathrm{~Hz}, \operatorname{ArCHCH}-\mathrm{C}\left(\mathrm{COOCH}_{3}\right)\right) ;{ }^{13} \mathrm{C} \mathrm{NMR}\left(100 \mathrm{MHz} / \mathrm{CDCl}_{3}\right) / \mathrm{ppm}, \delta=14.0$, 22.4, 33.7, 35.1, 51.7, 114.0, 120.6, 121.2, 129.4, 131.5, 138.2, 138.3, 148.7, 167.0; Found $[\mathrm{M}+\mathrm{H}]^{+}\left(\mathrm{C}_{18} \mathrm{H}_{22} \mathrm{NO}_{2}\right) \mathrm{m} / \mathrm{z}=284.1645$ (Calc. 284.1651); IR (ATR) v/cm-1:3340, 2922. 1689, $1591,1435,1285,754$.

4.2.11. Preparation of 2-((4-butylphenyl)amino)benzoic acid (21) [43]

Aqueous potassium hydroxide $(36 \mathrm{~mL}, 7.27 \mathrm{mmol})$ was added to $14(1.03 \mathrm{~g}, 3.63 \mathrm{mmol})$ in ethanol $(20 \mathrm{~mL})$ at room temperature and allowed to react according to the general ester hydrolysis procedure to yield $0.90 \mathrm{~g}$ (92\%) of 21 as a pale yellow solid; M.p. $147-148{ }^{\circ} \mathrm{C}$ (literature M.p. $\left.147-150{ }^{\circ} \mathrm{C}\right)$ [43]; ${ }^{1} \mathrm{H}$ NMR $\left(400 \mathrm{MHz} / \mathrm{CDCl}_{3}\right) / \mathrm{ppm}, \delta=0.95(\mathrm{t}, 3 \mathrm{H}, \mathrm{J}=8 \mathrm{~Hz}$, $\mathrm{CH}_{3} \mathrm{CH}_{2} \mathrm{CH}_{2} \mathrm{CH}_{2} \mathrm{Ar}$ ), 1.39 (sex., 2H, J = $8 \mathrm{~Hz},-\mathrm{CH}_{3} \mathrm{CH}_{2} \mathrm{CH}_{2} \mathrm{CH}_{2} \mathrm{Ar}$ ), 1.61 (quin., 2H, J = $8 \mathrm{~Hz}$, $-\mathrm{CH}_{3} \mathrm{CH}_{2} \mathrm{CH}_{2} \mathrm{CH}_{2} \mathrm{Ar}$ ), 2.61 (t, $2 \mathrm{H}, \mathrm{J}=8 \mathrm{~Hz},-\mathrm{CH}_{3} \mathrm{CH}_{2} \mathrm{CH}_{2} \mathrm{CH}_{2} \mathrm{Ar}$ ), 6.72 (app. t, $1 \mathrm{H}$, app $J=8$ Hz, HOOC-CHCHCHCHC-NH), 7.15 (app. d, 1H, HOOC-CHCHCHCHC-NH), 7.18 (app. s, 4H, $\mathrm{C}_{4} \mathrm{H}_{9}-\mathrm{ArCH}$ ), 7.33 (app. t, 1H, HOOC-CHCHCHCHC-NH), 8.01(app. d, 1H, HOOCCHCHCHCHC-NH), 9.25 (s, 1H,-NH); ${ }^{13} \mathrm{C}$ NMR (100 MHz/CDCl 3 )/ppm, $\delta=14.0,22.4,33.7$, $35.1,109.9,113.9,116.7,123.1,123.7,126.3,129.4,132.5,135.2,137.8,139.1,149.5$, 173.2; Found $[\mathrm{M}+\mathrm{H}]^{+}\left(\mathrm{C}_{17} \mathrm{H}_{22} \mathrm{NO}_{2}\right) \mathrm{m} / \mathrm{z}=270.1490$ (Calc. 270.1494); IR (ATR) v/cm ${ }^{-1}: 3333$, 3000-2480 (br), 2922, 1650, 1574, 1514, 1441, 1256, 746.

4.2.12. Preparation of 3-((4-butylphenyl)amino)benzoic acid (22) [44] 
Aqueous potassium hydroxide (14 $\mathrm{mL}, 2.76 \mathrm{mmol})$ was added to $17(0.39 \mathrm{~g}, 1.38 \mathrm{mmol})$ in ethanol $(7 \mathrm{~mL})$ at room temperature and allowed to react according to the general ester hydrolysis procedure to yield $0.30 \mathrm{~g}(81 \%)$ of 22 as a pale yellow solid; M.p. $146-148{ }^{\circ} \mathrm{C}$ (literature M.p. $\left.146-149{ }^{\circ} \mathrm{C}\right)[44] ;{ }^{1} \mathrm{H}$ NMR $\left(400 \mathrm{MHz} / \mathrm{CDCl}_{3}\right) / \mathrm{ppm}, \delta=0.94(\mathrm{t}, 3 \mathrm{H}, J=8 \mathrm{~Hz}$, $\mathrm{CH}_{3} \mathrm{CH}_{2} \mathrm{CH}_{2} \mathrm{CH}_{2} \mathrm{Ar}$ ), 1.38 (sex., $2 \mathrm{H}, \mathrm{J}=8 \mathrm{~Hz},-\mathrm{CH}_{3} \mathrm{CH}_{2} \mathrm{CH}_{2} \mathrm{CH}_{2} \mathrm{Ar}$ ), 1.60 (quin., $2 \mathrm{H}, \mathrm{J}=8 \mathrm{~Hz}$, $-\mathrm{CH}_{3} \mathrm{CH}_{2} \mathrm{CH}_{2} \mathrm{CH}_{2} \mathrm{Ar}$ ), 2.58 (t, $2 \mathrm{H}, J=8 \mathrm{~Hz},-\mathrm{CH}_{3} \mathrm{CH}_{2} \mathrm{CH}_{2} \mathrm{CH}_{2} \mathrm{Ar}$ ), 7.04 (app. d, 2H, app. $J=8$ Hz, HN-ArCHCH), 7.13 (app. d, 2H, app. J = $8 \mathrm{~Hz}, \mathrm{C}_{4} \mathrm{H}_{9}-\mathrm{ArCHCH}$ ), 7.23 (app. d, 1H, app. J $=8 \mathrm{~Hz}, \mathrm{HN}-\mathrm{CH}-\mathrm{C}(\mathrm{COOH})-\mathrm{CHCHCH}-\mathrm{NH}), 7.32$ (app. t, $1 \mathrm{H}, J=8 \mathrm{~Hz}, \mathrm{HN}-\mathrm{CH}-\mathrm{C}(\mathrm{COOH})-$ CHCHCH-NH), 7.60 (app. d, 1H, J = $8 \mathrm{~Hz}, \mathrm{HN}-\mathrm{CH}-\mathrm{C}(\mathrm{COOH})-\mathrm{CHCHCH}-\mathrm{NH}$ ), 7.72 (app. s, $1 \mathrm{H}, \mathrm{HN}-\mathrm{CH}-\mathrm{C}(\mathrm{COOH})-\mathrm{CHCHCH}-\mathrm{NH}) ;{ }^{13} \mathrm{C} \mathrm{NMR}\left(100 \mathrm{MHz} / \mathrm{CDCl}_{3}\right) / \mathrm{ppm}, \delta=14.0,22.4,33.8$, 35.0, 117.6, 119.5, 121.4, 121.7, 129.4, 129.5, 130.4, 137.1, 139.5, 144.5, 172.0; Found $[\mathrm{M}+\mathrm{H}]^{+}\left(\mathrm{C}_{17} \mathrm{H}_{22} \mathrm{NO}_{2}\right) \mathrm{m} / \mathrm{z}=270.1489$ (Calc. 270.1494); IR (ATR) v/cm ${ }^{-1}: 3386,3000-2500$ (br), 2928, 1687, 1520, 1288, 746.

\subsubsection{Preparation of 4-((4-butylphenyl)amino)benzoic acid (23) [45]}

Aqueous potassium hydroxide (18 $\mathrm{mL}, 3.53 \mathrm{mmol})$ was added to $19(0.50 \mathrm{~g}, 1.76 \mathrm{mmol})$ in ethanol $(9 \mathrm{~mL})$ at room temperature and allowed to react according to the general ester hydrolysis procedure to yield $0.41 \mathrm{~g}(86 \%)$ of 23 as a white solid; M.p. $138-140{ }^{\circ} \mathrm{C}$ (literature M.p. $\left.138-140{ }^{\circ} \mathrm{C}\right)[45] ;{ }^{1} \mathrm{H}$ NMR $\left(400 \mathrm{MHz} / \mathrm{CDCl}_{3}\right) / \mathrm{ppm}, \delta=0.94(\mathrm{t}, 3 \mathrm{H}, J=8 \mathrm{~Hz}$, $\mathrm{CH}_{3} \mathrm{CH}_{2} \mathrm{CH}_{2} \mathrm{CH}_{2} \mathrm{Ar}$ ), 1.39 (sex., $2 \mathrm{H}, J=8 \mathrm{~Hz},-\mathrm{CH}_{3} \mathrm{CH}_{2} \mathrm{CH}_{2} \mathrm{CH}_{2} \mathrm{Ar}$ ), 1.61 (quin., $2 \mathrm{H}, J=8 \mathrm{~Hz}$, $-\mathrm{CH}_{3} \mathrm{CH}_{2} \mathrm{CH}_{2} \mathrm{CH}_{2} \mathrm{Ar}$ ), 2.60 (t, $2 \mathrm{H}, \mathrm{J}=8 \mathrm{~Hz},-\mathrm{CH}_{3} \mathrm{CH}_{2} \mathrm{CH}_{2} \mathrm{CH}_{2} \mathrm{Ar}$ ), 6.00 (s, 1H, -NH), 6.94 (app. d, 2H, app. J $=12 \mathrm{~Hz}, \mathrm{HN}-\mathrm{ArCHCH}-\mathrm{CHCH}-\mathrm{C}(\mathrm{COOH})) ;), 7.11$ (app. d, 2H, app. J = $8 \mathrm{~Hz}, \mathrm{HN}-$ ArCHCH), 7.17 (app. d, 1H, app. $\left.J=8 \mathrm{~Hz}, \mathrm{C}_{4} \mathrm{H}_{9}-\mathrm{ArCHCH}\right), 7.96$ (app. d, 2H, J = $8 \mathrm{~Hz}$, $\operatorname{ArCHCH}-\mathrm{C}(\mathrm{COOH})) ;{ }^{13} \mathrm{C} \mathrm{NMR}\left(100 \mathrm{MHz} / \mathrm{CDCl}_{3}\right) / \mathrm{ppm}, \delta=14.0,22.4,33.7,35.1,113.9$, $119.4,121.6,129.4,132.3,137.9,138.6,149.5,171.5 ;$ Found $[\mathrm{M}+\mathrm{H}]^{+}\left(\mathrm{C}_{17} \mathrm{H}_{22} \mathrm{NO}_{2}\right) \mathrm{m} / \mathrm{z}=$ 
270.1489 (Calc. 270.1494); IR (ATR) v/cm-1: 3406, 3070-2545 (br), 2921, 1663, 1598, 1513, $1288,1175,755$.

\subsubsection{Preparation of mixed amine-phenol (24)}

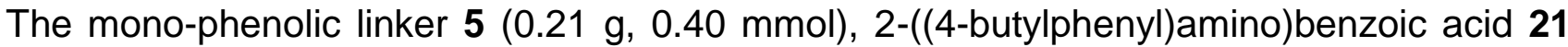
$(0.40 \mathrm{~g}, 1.49 \mathrm{mmol})$, DPTS $(60 \%)$ and DCC $(0.31 \mathrm{~g}, 1.49 \mathrm{mmol})$ were allowed to react according to the general mixed amine-phenol procedure. The crude product was purified by flash column chromatography on silica eluting with hexane/ethyl acetate $(90: 10)\left(R_{f}=0.25\right)$ to afford $0.25 \mathrm{~g}(82 \%)$ of 24 as a yellow oil; ${ }^{1} \mathrm{H} \mathrm{NMR}\left(400 \mathrm{MHz} / \mathrm{CDCl}_{3}\right) / \mathrm{ppm}, \delta=0.85(\mathrm{~m}, 6 \mathrm{H}$, $\left.-\mathrm{CH}_{2} \mathrm{CH}_{3}\right), 0.94$ (t, 3H, $\left.\mathrm{J}=8 \mathrm{~Hz},-\mathrm{CH}_{3} \mathrm{CH}_{2} \mathrm{CH}_{2} \mathrm{CH}_{2} \mathrm{Ar}\right), 1.25-1.38\left(\mathrm{~m}, 13 \mathrm{H},-\mathrm{CH}_{2} \mathrm{CH}_{3},-\mathrm{CH}_{3}\right.$ and $\mathrm{CH}_{3} \mathrm{CH}_{2} \mathrm{CH}_{2} \mathrm{CH}_{2} \mathrm{Ar}$ ), 1.42 (s, $18 \mathrm{H}, \mathrm{CH}_{3}$ tert-butyl), 1.60 (m, 3H, $-\mathrm{CH}_{3} \mathrm{CH}_{2} \mathrm{CH}_{2} \mathrm{CH}_{2} \mathrm{Ar}$ and $\left.\mathrm{CH}\left(\mathrm{CH}_{2} \mathrm{CH}_{3}\right)\right), 2.61\left(\mathrm{~m}, 4 \mathrm{H}, \mathrm{Ph}-\mathrm{CH}_{2} \mathrm{CH}_{2}-\mathrm{COO}\right.$ - and $\left.-\mathrm{C}_{3} \mathrm{H}_{7} \mathrm{CH}_{2} \mathrm{Ar}\right), 2.85(\mathrm{t}, 2 \mathrm{H}, \mathrm{J}=8 \mathrm{~Hz}, \mathrm{Ph}-$ $\left.\mathrm{CH}_{2} \mathrm{CH}_{2}-\mathrm{COO}-\right), 4.08$ (m, $\left.2 \mathrm{H}, \mathrm{COO}-\mathrm{CH}_{2}-\mathrm{CH}\left(\mathrm{CH}_{2} \mathrm{CH}_{3}\right)-\mathrm{C}_{4} \mathrm{H}_{9}\right), 4.37$ (s, $\left.2 \mathrm{H}, \mathrm{COO}-\mathrm{CH}_{2}-\mathrm{C}\right), 4.42$ (s, 2H, COO- $\mathrm{CH}_{2} \mathrm{C}$ ), 5.07 (s, 1H, -OH), 6.67 (app. t, 1H, -OOC-CHCHCHCHC-NH), 6.97 (s, 2H, Ph- $\mathrm{CH}$ ), 7.15 (m, 5H, $\mathrm{C}_{4} \mathrm{H}_{9}-\mathrm{ArCH}$ and -OOC-CHCHCHCHC-NH), 7.29 (app. d, $1 \mathrm{H}, \mathrm{J}=8$ $\mathrm{Hz},-\mathrm{OOC}-\mathrm{CHCHCHCHC}-\mathrm{NH}$ ), 7.85 (app. d, 1H, $J=8 \mathrm{~Hz}$, -OOC-CHCHCHCHC-NH), 9.31 (s, $1 \mathrm{H},-\mathrm{NH}) ;{ }^{13} \mathrm{C} \mathrm{NMR}\left(100 \mathrm{MHz} / \mathrm{CDCl}_{3}\right) / \mathrm{ppm}, \delta=10.9,14.0,18.0,22.4,22.9,23.7,28.9$, $30.3,30.9,33.7,34.3,35.1,36.2,38.7,46.6,65.8,67.5,110.7,113.8,116.7,123.3,124.7$, $129.3,130.8,131.4,135.9,137.9,138.8,148.8,152.2,167.8,172.7,173.0$; Found $[\mathrm{M}+\mathrm{Na}]^{+}$ $\left(\mathrm{C}_{47} \mathrm{H}_{67} \mathrm{NO} \mathrm{O}_{7} \mathrm{Na}\right.$ ) $\mathrm{m} / \mathrm{z}=780.4810$ (Calc. 780.4815); IR (ATR) v/cm ${ }^{-1}: 3636,3324,2960,1731$, $1516,1225,1134,748$.

\subsubsection{Preparation of mixed amine-phenol (25)}

The mono-phenolic linker 5 (0.61 g, 1.20 mmol), 3-((4-butylphenyl)amino)benzoic acid 22 $(0.48 \mathrm{~g}, 1.80 \mathrm{mmol})$, DPTS $(60 \%)$ and DCC $(0.37 \mathrm{~g}, 1.80 \mathrm{mmol})$ were allowed to react according to the general mixed amine-phenol procedure. The crude product was purified by 
flash column chromatography on silica eluting with hexane/ethyl acetate (90:10) $\left(R_{f}=0.23\right)$ to afford $0.75 \mathrm{~g}(82 \%)$ of 25 as a dark yellow oil; ${ }^{1} \mathrm{H} \mathrm{NMR}\left(400 \mathrm{MHz} / \mathrm{CDCl}_{3}\right) / \mathrm{ppm}, \delta=0.84$ (m, $6 \mathrm{H},-\mathrm{CH}_{2} \mathrm{CH}_{3}$ ), 0.93 (t, 3H, J=8 Hz, $-\mathrm{CH}_{3} \mathrm{CH}_{2} \mathrm{CH}_{2} \mathrm{CH}_{2} \mathrm{Ar}$ ), 1.23-1.39 (m, 13H, $-\mathrm{CH}_{2} \mathrm{CH}_{3}$, $-\mathrm{CH}_{3}$ and $\mathrm{CH}_{3} \mathrm{CH}_{2} \mathrm{CH}_{2} \mathrm{CH}_{2} \mathrm{Ar}$ ), 1.42 (s, $18 \mathrm{H}, \mathrm{CH}_{3}$ tert-butyl), 1.57 (m, 3H, $-\mathrm{CH}_{3} \mathrm{CH}_{2} \mathrm{CH}_{2} \mathrm{CH}_{2} \mathrm{Ar}$ and $\left.-\mathrm{CH}\left(\mathrm{CH}_{2} \mathrm{CH}_{3}\right)\right), 2.59\left(\mathrm{~m}, 4 \mathrm{H}, \mathrm{Ph}-\mathrm{CH}_{2} \mathrm{CH}_{2}-\mathrm{COO}\right.$ - and $\left.-\mathrm{C}_{3} \mathrm{H}_{7} \mathrm{CH}_{2} \mathrm{Ar}\right), 2.84(\mathrm{t}, 2 \mathrm{H}, J=8 \mathrm{~Hz}$, Ph- $\left.\mathrm{CH}_{2} \mathrm{CH}_{2}-\mathrm{COO}-\right)$, 4.05 (m, 2H, $\left.\mathrm{COO}-\mathrm{CH}_{2}-\mathrm{CH}\left(\mathrm{CH}_{2} \mathrm{CH}_{3}\right)-\mathrm{C}_{4} \mathrm{H}_{9}\right)$, 4.34 (s, $2 \mathrm{H}, \mathrm{COO}-\mathrm{CH}_{2}-\mathrm{C}$ ), $4.43\left(\mathrm{~s}, 2 \mathrm{H}, \mathrm{COO}-\mathrm{CH}_{2}-\mathrm{C}\right), 5.07(\mathrm{~s}, 1 \mathrm{H},-\mathrm{OH}), 5.75(\mathrm{~s}, 1 \mathrm{H},-\mathrm{NH}), 6.97(\mathrm{~s}, 2 \mathrm{H}, \mathrm{Ph}-\mathrm{CH}), 7.02$ (app. d, $1 \mathrm{H}$, app. $J=8 \mathrm{~Hz}, \operatorname{ArCH}-\mathrm{C}\left(\mathrm{C}_{4} \mathrm{H}_{9}\right)$ ), 7.11 (app. d, 2H, app. $J=8 \mathrm{~Hz}, \mathrm{ArCH}-\mathrm{CNH}$ ), 7.21 (app. d, 2H, app. J = $8 \mathrm{~Hz}, \mathrm{HN}-\mathrm{CH}-\mathrm{C}(\mathrm{COO}-)-\mathrm{CHCHCH}-), 7.28$ (m, 1H, HN-CH-C(COO)- $\mathrm{CHCHCH}-), 7.46$ (app. d, 1H, J = $8 \mathrm{~Hz}, \mathrm{HN}-\mathrm{CH}-\mathrm{C}(\mathrm{COO}-)-\mathrm{CHCHCH}-), 7.60(\mathrm{~m}, 1 \mathrm{H}, \mathrm{HN}-\mathrm{CH}-$ $\mathrm{C}(\mathrm{COO}-)) ;{ }^{13} \mathrm{C} \mathrm{NMR}\left(100 \mathrm{MHz} / \mathrm{CDCl}_{3}\right) / \mathrm{ppm}, \delta=11.0,14.0,17.9,22.4,22.9,23.7,28.9,30.3$, 30.9, 33.8, 34.3, 35.0, 36.2, 38.7, 46.6, 65.7, 66.1, 67.4, 117.3, 119.4, 120.7, 121.0, 124.7, $129.4,130.8,130.9,136.0,144.4,152.2,166.1,172.7,172.9$; Found $[\mathrm{M}+\mathrm{H}]^{+}\left(\mathrm{C}_{47} \mathrm{H}_{68} \mathrm{NO}_{7}\right)$ $\mathrm{m} / \mathrm{z}=758.4961$ (Calc. 758.4996); IR (ATR) $v / \mathrm{cm}^{-1}: 3633,3381,2955,1725,1213,749$.

\subsubsection{Preparation of mixed amine-phenol (26)}

The mono-phenolic linker 5 (0.50 g, 0.99 mmol), 4-((4-butylphenyl)amino)benzoic acid 23 $(0.40 \mathrm{~g}, 1.49 \mathrm{mmol})$, DPTS (60\%) and DCC $(0.31 \mathrm{~g}, 1.49 \mathrm{mmol})$ were allowed to react according to the general mixed amine-phenol procedure. The crude product was purified by flash column chromatography on silica eluting with hexane/ethyl acetate $(80: 20)\left(R_{f}=0.33\right)$ to afford $0.65 \mathrm{~g}(86 \%)$ of 26 as an orange oil; ${ }^{1} \mathrm{H} \mathrm{NMR}\left(400 \mathrm{MHz} / \mathrm{CDCl}_{3}\right) / \mathrm{ppm}, \delta=0.84(\mathrm{~m}$, $\left.6 \mathrm{H},-\mathrm{CH}_{2} \mathrm{CH}_{3}\right), 0.94\left(\mathrm{t}, 3 \mathrm{H}, \mathrm{J}=8 \mathrm{~Hz},-\mathrm{CH}_{3} \mathrm{CH}_{2} \mathrm{CH}_{2} \mathrm{CH}_{2} \mathrm{Ar}\right), 1.24-1.21\left(\mathrm{~m}, 11 \mathrm{H},-\mathrm{CH}_{2} \mathrm{CH}_{3}\right.$, $\left.\mathrm{CH}_{3}\right), 1.36\left(\mathrm{~m}, 2 \mathrm{H}, \mathrm{CH}_{3} \mathrm{CH}_{2} \mathrm{CH}_{2} \mathrm{CH}_{2} \mathrm{Ar}\right), 1.42\left(\mathrm{~s}, 18 \mathrm{H}, \mathrm{CH}_{3}\right.$ tert-butyl), $1.58(\mathrm{~m}, 3 \mathrm{H},-$ $\mathrm{CH}_{3} \mathrm{CH}_{2} \mathrm{CH}_{2} \mathrm{CH}_{2} \mathrm{Ar}$ and $\left.-\mathrm{CH}\left(\mathrm{CH}_{2} \mathrm{CH}_{3}\right)\right), 2.60\left(\mathrm{~m}, 4 \mathrm{H}, \mathrm{Ph}-\mathrm{CH}_{2} \mathrm{CH}_{2}-\mathrm{COO}\right.$ - and $-\mathrm{C}_{3} \mathrm{H}_{7} \mathrm{CH}_{2} \mathrm{Ar}$ ), 2.84 (t, $\left.2 \mathrm{H}, \mathrm{J}=8 \mathrm{~Hz}, \mathrm{Ph}-\mathrm{CH}_{2} \mathrm{CH}_{2}-\mathrm{COO}-\right), 4.06$ (m, 2H, $\left.\mathrm{COO}-\mathrm{CH}_{2}-\mathrm{CH}\left(\mathrm{CH}_{2} \mathrm{CH}_{3}\right)-\mathrm{C}_{4} \mathrm{H}_{9}\right), 4.34$ (s, $2 \mathrm{H}, \mathrm{COO}-\mathrm{CH}_{2}-\mathrm{C}$ ), 4.40 (s, 2H, COO- $\mathrm{CH}_{2}-\mathrm{C}$ ), 5.07 (s, 1H, -OH), 5.97 (s, 1H, -NH), 6.91 (app. 
d, $2 \mathrm{H}$, app. $\mathrm{J}=8 \mathrm{~Hz}, \mathrm{NH}-\mathrm{ArC}-\mathrm{CH}$ ), 6.97 (s, 2H, Ph-CH), 7.06 (app. d, 2H, app. $\mathrm{J}=8 \mathrm{~Hz}$, $\operatorname{ArCH}-\mathrm{C}\left(\mathrm{C}_{4} \mathrm{H}_{9}\right)$ ), 7.15 (app. d, 2H, app. $J=8 \mathrm{~Hz}, \mathrm{ArCH}-\mathrm{CNH}$ ), 7.84 (app. d, 2H, app. J = $8 \mathrm{~Hz}$, $\operatorname{ArCH}-\mathrm{C}(\mathrm{COO}-)) ;{ }^{13} \mathrm{C} \mathrm{NMR}\left(100 \mathrm{MHz} / \mathrm{CDCl}_{3}\right) / \mathrm{ppm}, \delta=11.0,14.0,14.1,17.9,22.3,22.9,23.7$, $28.9,30.3,30.9,33.7,34.3,35.0,36.2,38.7,46.7,65.6,65.7,67.4,114.0,119.9,121.3$, $124.7,129.4,130.9,131.6,135.9,138.1,138.4,149.0,152.2,165.9,172.8,173.1$; Found $\left[\mathrm{M}+\mathrm{Na}^{+}\left(\mathrm{C}_{47} \mathrm{H}_{67} \mathrm{NO} 7 \mathrm{Na}\right) \mathrm{m} / \mathrm{z}=780.4810\right.$ (Calc. 780.4815); IR (ATR) $\mathrm{v} / \mathrm{cm}^{-1}: 3636,3361$, $2960,1715,1598,1517,1170,754$.

\subsubsection{Preparation of bis(diphenylamine) (27)}

The first generation hydroxyl linker $6(0.20 \mathrm{~g}, 0.91 \mathrm{mmol}), 2-((4-$ butylphenyl)amino)benzoic acid 21 (0.56 g, $2.09 \mathrm{mmol})$, DPTS (60\%) and DCC (0.43 g, $2.09 \mathrm{mmol})$ were allowed to react according to the general bis(diphenylamine) procedure. The crude product was purified by flash column chromatography on silica eluting with chloroform/hexane $(70: 30)\left(R_{f}=0.56\right)$ to afford $0.56 \mathrm{~g}(82 \%)$ of 27 as a yellow oil; ${ }^{1} \mathrm{H} \mathrm{NMR}\left(400 \mathrm{MHz} / \mathrm{CDCl}_{3}\right) / \mathrm{ppm}, \delta=0.83(\mathrm{~m}, 6 \mathrm{H}$, $-\mathrm{CH}_{2} \mathrm{CH}_{3}$ ), 0.92 (t, $6 \mathrm{H}, J=8 \mathrm{~Hz},-\mathrm{CH}_{3} \mathrm{CH}_{2} \mathrm{CH}_{2} \mathrm{CH}_{2} \mathrm{Ar}$ ), 1.21-1.40 (m, $12 \mathrm{H},-\mathrm{CH}_{2} \mathrm{CH}_{3}$ and $\mathrm{CH}_{3} \mathrm{CH}_{2} \mathrm{CH}_{2} \mathrm{CH}_{2} \mathrm{Ar}$ ), 1.46 (s, 3H, $\left.-\mathrm{CH}_{3}\right), 1.60$ (m, $5 \mathrm{H},-\mathrm{CH}_{3} \mathrm{CH}_{2} \mathrm{CH}_{2} \mathrm{CH}_{2} \mathrm{Ar}$ and $-\mathrm{CH}\left(\mathrm{CH}_{2} \mathrm{CH}_{3}\right)$ ), $2.59\left(\mathrm{t}, 4 \mathrm{H}, J=8 \mathrm{~Hz},-\mathrm{CH}_{3} \mathrm{CH}_{2} \mathrm{CH}_{2} \mathrm{CH}_{2} \mathrm{Ar}\right), 4.10\left(\mathrm{~m}, 2 \mathrm{H}, \mathrm{COO}-\mathrm{CH}_{2}-\mathrm{CH}\left(\mathrm{CH}_{2} \mathrm{CH}_{3}\right)-\mathrm{C}_{4} \mathrm{H}_{9}\right), 4.56$ (s, 4H, COO- $\mathrm{CH}_{2}-\mathrm{C}$ ), 6.66 (app. t, 2H, app. J = $8 \mathrm{~Hz}$, -OOC-CHCHCHCHC-NH), 7.15 (m, $10 \mathrm{H}, \mathrm{C}_{4} \mathrm{H}_{9}-\mathrm{ArCH}$ and $\left.-\mathrm{OOC}-\mathrm{CHCHCHCHC}-\mathrm{NH}\right), 7.30$ (m, 2H, -OOC-CHCHCHCHC-NH), 7.88 (app. d, 2H, app. J=8 Hz, -OOC-CHCHCHCHC-NH), $9.32(\mathrm{~s}, 2 \mathrm{H},-\mathrm{NH}) ;{ }^{13} \mathrm{C}$ NMR $(100$ $\left.\mathrm{MHz} / \mathrm{CDCl}_{3}\right) / \mathrm{ppm}, \delta=10.9,14.0,22.4,22.9,23.7,28.9,30.3,33.7,35.1,46.9,66.0,67.6$, $110.7,113.8,116.6,123.3,126.2,129.3,131.4,134.4,138.0,138.7,148.8,167.8,173.1$; Found $[\mathrm{M}+\mathrm{Na}]^{+}\left(\mathrm{C}_{47} \mathrm{H}_{60} \mathrm{~N}_{2} \mathrm{O}_{6} \mathrm{Na}\right) \mathrm{m} / \mathrm{z}=771.4344$ (Calc. 771.4349); IR (ATR) $\mathrm{v} / \mathrm{cm}^{-1}: 3320$, $2960,1683,1514,1216,1074,745$.

4.2.18. Preparation of bis(diphenylamine) (28) 
The first generation hydroxyl linker 6 (0.17 g, $0.74 \mathrm{mmol})$, 3-((4-butylphenyl)amino)benzoic acid $22(0.46 \mathrm{~g}, 1.70 \mathrm{mmol})$, DPTS (60\%) and DCC (0.35 g, $1.70 \mathrm{mmol})$ were allowed to react according to the general bis(diphenylamine) procedure. The crude product was purified by flash column chromatography on silica eluting with hexane/ethyl acetate $(80: 20)\left(R_{f}=0.50\right)$ to afford $0.42 \mathrm{~g}(75 \%)$ of 28 as a pale yellow oil; ${ }^{1} \mathrm{H}$ NMR $\left(400 \mathrm{MHz} / \mathrm{CDCl}_{3}\right) / \mathrm{ppm}, \delta=0.80(\mathrm{~m}$, $\left.6 \mathrm{H},-\mathrm{CH}_{2} \mathrm{CH}_{3}\right), 0.93\left(\mathrm{t}, 6 \mathrm{H}, \mathrm{J}=8 \mathrm{~Hz},-\mathrm{CH}_{3} \mathrm{CH}_{2} \mathrm{CH}_{2} \mathrm{CH}_{2} \mathrm{Ar}\right), 1.19-1.37\left(\mathrm{~m}, 12 \mathrm{H},-\mathrm{CH}_{2} \mathrm{CH}_{3}\right.$ and $\left.\mathrm{CH}_{3} \mathrm{CH}_{2} \mathrm{CH}_{2} \mathrm{CH}_{2} \mathrm{Ar}\right), 1.40$ (s, 3H, $\left.-\mathrm{CH}_{3}\right), 1.58\left(\mathrm{~m}, 5 \mathrm{H},-\mathrm{CH}_{3} \mathrm{CH}_{2} \mathrm{CH}_{2} \mathrm{CH}_{2} \mathrm{Ar}\right.$ and $\left.-\mathrm{CH}\left(\mathrm{CH}_{2} \mathrm{CH}_{3}\right)\right)$, $2.56\left(\mathrm{t}, 4 \mathrm{H}, \mathrm{J}=8 \mathrm{~Hz},-\mathrm{C}_{3} \mathrm{H}_{7} \mathrm{CH}_{2} \mathrm{Ar}\right), 4.05\left(\mathrm{~m}, 2 \mathrm{H}, \mathrm{COO}-\mathrm{CH}_{2}-\mathrm{CH}\left(\mathrm{CH}_{2} \mathrm{CH}_{3}\right)-\mathrm{C}_{4} \mathrm{H}_{9}\right), 4.55(\mathrm{~s}, 4 \mathrm{H}$, COO- $\mathrm{CH}_{2}-\mathrm{C}$ ), 5.74 (s, 2H, -NH), 7.00 (app. d, 4H, app. J = $8 \mathrm{~Hz}, \operatorname{ArCH}-\mathrm{C}\left(\mathrm{C}_{4} \mathrm{H}_{9}\right)$ ), 7.10 (app. d, 4H, app. $J=8 \mathrm{~Hz}, \mathrm{ArCH}-\mathrm{CNH}), 7.18\left(\mathrm{~m}, 2 \mathrm{H}, \mathrm{HN}-\mathrm{CH}-\mathrm{C}(\mathrm{COO}-)-\mathrm{CHCHCH}^{-}\right), 7.25$ (m, 2H, $\mathrm{HN}-\mathrm{CH}-\mathrm{C}(\mathrm{COO}-)-\mathrm{CHCHCH}-), 7.46$ (app. d, 2H, app. J = $8 \mathrm{~Hz}, \mathrm{HN}-\mathrm{CH}-\mathrm{C}(\mathrm{COO}-)-\mathrm{CHCHCH}-)$, $7.60(\mathrm{~m}, 2 \mathrm{H}, \mathrm{HN}-\mathrm{CH}-\mathrm{C}(\mathrm{COO}-)) ;{ }^{13} \mathrm{C}$ NMR $\left(100 \mathrm{MHz} / \mathrm{CDCl}_{3}\right) / \mathrm{ppm}, \delta=11.0,14.0,18.1,22.3$, 22.9, 23.7, 28.9, 30.3, 33.8, 35.0, 38.7, 46.8, 66.3, 67.5, 117.3, 118.9, 119.4, 120.6, 121.0, 126.3, 129.3, 130.8, 136.9, 139.6, 144.4, 166.1, 172.9; Found $[\mathrm{M}+\mathrm{Na}]^{+}\left(\mathrm{C}_{47} \mathrm{H}_{60} \mathrm{~N}_{2} \mathrm{O}_{6} \mathrm{Na}\right) \mathrm{m} / \mathrm{z}$ $=771.4343$ (Calc. 771.4349); IR (ATR) $v / \mathrm{cm}^{-1}: 3376,2960,1721,1515,1208,1103,747$.

\subsubsection{Preparation of bis(diphenylamine) (29)}

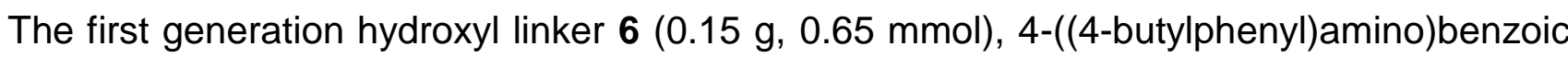
acid $23(0.40 \mathrm{~g}, 1.49 \mathrm{mmol})$, DPTS (60\%) and DCC (0.31 g, $1.49 \mathrm{mmol})$ were allowed to react according to the general bis(diphenylamine) procedure. The crude product was purified by flash column chromatography on silica eluting with hexane/ethyl acetate $(80: 20)\left(R_{f}=\right.$ $0.53)$ to afford $0.44 \mathrm{~g}(90 \%)$ of 29 as a pale orange oil; ${ }^{1} \mathrm{H} \mathrm{NMR}(400 \mathrm{MHz} / \mathrm{CDCl} 3) / \mathrm{ppm}, \delta=$ $0.82\left(\mathrm{~m}, 6 \mathrm{H},-\mathrm{CH}_{2} \mathrm{CH}_{3}\right), 0.94\left(\mathrm{t}, 6 \mathrm{H}, \mathrm{J}=8 \mathrm{~Hz},-\mathrm{CH}_{3} \mathrm{CH}_{2} \mathrm{CH}_{2} \mathrm{CH}_{2} \mathrm{Ar}\right), 1.20-1.38(\mathrm{~m}, 15 \mathrm{H}$, $\mathrm{CH}_{2} \mathrm{CH}_{3}$, $-\mathrm{CH}_{3}$, and $\left.\mathrm{CH}_{3} \mathrm{CH}_{2} \mathrm{CH}_{2} \mathrm{CH}_{2} \mathrm{Ar}\right), \quad 1.61$ (m, 5H, $-\mathrm{CH}_{3} \mathrm{CH}_{2} \mathrm{CH}_{2} \mathrm{CH}_{2} \mathrm{Ar}$ and $\left.\mathrm{CH}\left(\mathrm{CH}_{2} \mathrm{CH}_{3}\right)\right), 2.59\left(\mathrm{t}, 4 \mathrm{H}, J=8 \mathrm{~Hz},-\mathrm{CH}_{3} \mathrm{CH}_{2} \mathrm{CH}_{2} \mathrm{CH}_{2} \mathrm{Ar}\right), 4.08\left(\mathrm{~m}, 2 \mathrm{H}, \mathrm{COO}-\mathrm{CH}_{2}\right.$ $\left.\mathrm{CH}\left(\mathrm{CH}_{2} \mathrm{CH}_{3}\right)-\mathrm{C}_{4} \mathrm{H}_{9}\right), 4.52$ (s, 4H, COO- $\left.\mathrm{CH}_{2}-\mathrm{C}\right), 5.96$ (s, 2H, -NH), 6.90 (app. d, 4H, app. J = 
$8 \mathrm{~Hz}, \mathrm{ArCH}-\mathrm{CNH}$ ), 7.08 (app. d, 4H, app. J $=8 \mathrm{~Hz}, \mathrm{ArCH}-\mathrm{C}\left(\mathrm{C}_{4} \mathrm{H}_{9}\right)$ ), 7.15 (app. d, 4H, app. J $=8 \mathrm{~Hz}, \operatorname{ArCH}-\mathrm{CNH}$ ), 7.85 (app. d, 4H, app. $J=8 \mathrm{~Hz} \operatorname{ArCH}-\mathrm{C}(\mathrm{COO}-)$ ); ${ }^{13} \mathrm{C}$ NMR (100 $\left.\mathrm{MHz} / \mathrm{CDCl}_{3}\right) / \mathrm{ppm}, \delta=10.9,14.0,18.1,22.3,22.9,23.7,28.9,30.3,33.7,35.0,38.7,47.0$, $65.9,67.4,114.0,120.0,121.2,129.4,131.6,138.1,138.1,148.9,165.9,173.2$; Found $[\mathrm{M}+\mathrm{Na}]^{+}\left(\mathrm{C}_{47} \mathrm{H}_{60} \mathrm{~N}_{2} \mathrm{O}_{6} \mathrm{Na}\right) \mathrm{m} / \mathrm{z}=771.4344$ (Calc. 771.4349); IR (ATR) $\mathrm{v} / \mathrm{cm}^{-1}: 3345,2960$, 1696, 1596, 1514, 1265, 1168, 1100, 755.

4.2.20. Preparation of methyl 3-(3-((4-butylphenyl)amino)phenyl)propanoate (30)

Methyl 3-(3-bromophenyl)propanoate $31(1.00 \mathrm{~g}, 4.11 \mathrm{mmol})$ and 4-butylaniline 16 (0.78 mL, $4.93 \mathrm{mmol})$ were added to $\mathrm{Pd}(\mathrm{OAc})_{2}(0.02 \mathrm{~g}, 0.08 \mathrm{mmol})$, BINAP $(0.10 \mathrm{~g}, 0.16 \mathrm{mmol})$ and $\mathrm{Cs}_{2} \mathrm{CO}_{3}(1.87 \mathrm{~g}, 5.75 \mathrm{mmol})$ and were allowed to react according to the general BuchwaldHartwig amination procedure. The crude residue was purified by flash column chromatography on silica eluting with hexane/ethyl acetate $(80: 20)\left(R_{f}=0.41\right)$ to afford $0.43 \mathrm{~g}$ (34\%) of 30 as an orange oil; ${ }^{1} \mathrm{H}$ NMR $\left(400 \mathrm{MHz} / \mathrm{CDCl}_{3}\right) / \mathrm{ppm}, \delta=0.93(\mathrm{t}, 3 \mathrm{H}, J=8 \mathrm{~Hz}$, $\mathrm{CH}_{3} \mathrm{CH}_{2} \mathrm{CH}_{2} \mathrm{CH}_{2} \mathrm{Ar}$ ), 1.36 (sex., $2 \mathrm{H}, J=8 \mathrm{~Hz},-\mathrm{CH}_{3} \mathrm{CH}_{2} \mathrm{CH}_{2} \mathrm{CH}_{2} \mathrm{Ar}$ ), 1.58 (quin., $2 \mathrm{H}, J=8 \mathrm{~Hz}$, $-\mathrm{CH}_{3} \mathrm{CH}_{2} \mathrm{CH}_{2} \mathrm{CH}_{2} \mathrm{Ar}$ ), 2.56 (t, $2 \mathrm{H}, J=8 \mathrm{~Hz},-\mathrm{CH}_{3} \mathrm{CH}_{2} \mathrm{CH}_{2} \mathrm{CH}_{2} \mathrm{Ar}$ ), 2.61 (t, $2 \mathrm{H}, J=8 \mathrm{~Hz}$, ArC$\mathrm{CH}_{2} \mathrm{CH}_{2}-\mathrm{COOCH}_{3}$ ), 2.89 (t, $\left.2 \mathrm{H}, \mathrm{J}=8 \mathrm{~Hz}, \mathrm{ArC}-\mathrm{CH}_{2} \mathrm{CH}_{2}-\mathrm{COOCH}_{3}\right), 3.67$ (s, 3H,- $\mathrm{COOCH}_{3}$ ), $5.60(\mathrm{~s}, 1 \mathrm{H},-\mathrm{NH}), 6.71$ ( app. d, app. $J=8 \mathrm{~Hz}, 2 \mathrm{H}, \mathrm{HN}-\mathrm{CH}-\mathrm{C}\left(\mathrm{CH}_{2} \mathrm{CH}_{2}\left(\mathrm{COOCH}_{3}\right)\right)-\mathrm{CHCHCH}-$ ), 6.86 (app. d, app. J = $8 \mathrm{~Hz}, 2 \mathrm{H}, \mathrm{HN}-\mathrm{CH}-\mathrm{C}\left(\mathrm{CH}_{2} \mathrm{CH}_{2}\left(\mathrm{COOCH}_{3}\right)\right.$ )- $\mathrm{CHCHCH}$ ), 7.00 (app. d, 2H, app. J = $8 \mathrm{~Hz}, \mathrm{HN}-\mathrm{ArCHCH}$ ), 7.09 (app. d, 2H, app. J = $\left.8 \mathrm{~Hz}, \mathrm{C}_{4} \mathrm{H}_{9}-\mathrm{ArCHCH}\right), 7.14$ (app. d, $1 \mathrm{H}$, app. $\left.J=8 \mathrm{~Hz}, \mathrm{HN}-\mathrm{CH}-\mathrm{C}\left(\mathrm{COOCH}_{3}\right)-\mathrm{CHCHCH}-\mathrm{NH}\right) ;{ }^{13} \mathrm{C} \mathrm{NMR}\left(100 \mathrm{MHz} / \mathrm{CDCl}_{3}\right) / \mathrm{ppm}$, $\delta=14.0,22.4,31.0,33.8,35.0,35.7,51.7,114.8,116.7,118.8,120.2,129.2,129.4,136.1$, 140.4, 141.8, 144.1, 173.4; Found $[\mathrm{M}+\mathrm{H}]^{+}\left(\mathrm{C}_{20} \mathrm{H}_{26} \mathrm{NO}_{2}\right) \mathrm{m} / \mathrm{z}=312.1958$ (Calc. 312.1964); IR (ATR) $v / \mathrm{cm}^{-1}: 3380,2925,1725,1591,1514,1166,778$.

4.2.21. Preparation of ethyl 2-(4-((4-butylphenyl)amino)phenyl)acetate (32) 
Ethyl 2-(4-(bromophenyl)acetate $33(0.50 \mathrm{~g}, 2.06 \mathrm{mmol})$ and 4-butylaniline 16 (0.39 mL, 2.47 mmol) were added to $\mathrm{Pd}(\mathrm{OAc})_{2}(0.01 \mathrm{~g}, 0.04 \mathrm{mmol})$, BINAP $(0.05 \mathrm{~g}, 0.08 \mathrm{mmol})$ and $\mathrm{Cs}_{2} \mathrm{CO}_{3}$ $(0.94 \mathrm{~g}, 2.88 \mathrm{mmol})$ and were allowed to react according to the general Buchwald-Hartwig amination procedure. The crude residue was purified by flash column chromatography on silica eluting with hexane/ethyl acetate $(85: 15)\left(R_{f}=0.33\right)$ to afford $0.43 \mathrm{~g}(67 \%)$ of 32 as an orange oil; ${ }^{1} \mathrm{H}$ NMR (400 MHz/CDCl 3$) / p p m, \delta=0.92\left(\mathrm{t}, 3 \mathrm{H}, J=8 \mathrm{~Hz},-\mathrm{CH}_{3} \mathrm{CH}_{2} \mathrm{CH}_{2} \mathrm{CH}_{2} \mathrm{Ar}\right.$ ), 1.25 (t, 3H, J=8 Hz, ArC- $\mathrm{CH}_{2}-\mathrm{COOCH}_{2} \mathrm{CH}_{3}$ ), 1.35 (sex., $2 \mathrm{H}, J=8 \mathrm{~Hz},-\mathrm{CH}_{3} \mathrm{CH}_{2} \mathrm{CH}_{2} \mathrm{CH}_{2} \mathrm{Ar}$ ), 1.57 (quin, $2 \mathrm{H}, J=8 \mathrm{~Hz},-\mathrm{CH}_{3} \mathrm{CH}_{2} \mathrm{CH}_{2} \mathrm{CH}_{2} \mathrm{Ar}$ ), 2.55 (t, $2 \mathrm{H}, J=8 \mathrm{~Hz},-\mathrm{CH}_{3} \mathrm{CH}_{2} \mathrm{CH}_{2} \mathrm{CH}_{2} \mathrm{Ar}$ ), $3.53\left(\mathrm{~s}, 2 \mathrm{H}, \mathrm{ArC}-\mathrm{CH}_{2}-\mathrm{COOCH}_{2} \mathrm{CH}_{3}\right), 4.14$ (q, $\left.2 \mathrm{H}, \mathrm{J}=8 \mathrm{~Hz}, \mathrm{ArC}-\mathrm{CH}_{2}-\mathrm{COOCH}_{2} \mathrm{CH}_{3}\right), 5.62$ (s, $1 \mathrm{H},-\mathrm{NH}), 6.97\left(\mathrm{~m}, 4 \mathrm{H}, \mathrm{HN}-\mathrm{ArCH}_{4}-\mathrm{CH}_{2}\left(\mathrm{COOCH}_{2} \mathrm{CH}_{3}\right)\right), 7.00$ (app. d, $2 \mathrm{H}$, app. J = $8 \mathrm{~Hz}, \mathrm{HN}-$ $\operatorname{ArCHCH}), 7.07$ (app. d, 2H, app. $\left.J=8 \mathrm{~Hz}, \mathrm{C}_{4} \mathrm{H}_{9}-\mathrm{ArCHCH}\right) ;{ }^{13} \mathrm{C} \mathrm{NMR}\left(100 \mathrm{MHz} / \mathrm{CDCl}_{3}\right) / \mathrm{ppm}$, $\delta=14.0,14.3,22.4,33.9,35.0,40.7,60.8,117.1,118.6,125.8,129.2,130.1,136.0,140.6$, 142.9, 172.1; Found $[\mathrm{M}+\mathrm{H}]^{+}\left(\mathrm{C}_{20} \mathrm{H}_{26} \mathrm{NO}_{2}\right) \mathrm{m} / \mathrm{z}=312.1958$ (Calc. 312.1964$) ; \mathrm{IR}(\mathrm{ATR}) \mathrm{v} / \mathrm{cm}^{-1}$ : 3385, 2928, 1725, 1608, 1514, 1308, 1140, 750.

\subsubsection{Preparation of methyl 3-(4-((4-butylphenyl)amino)phenyl)propanoate (34)}

Methyl 3-(4-(bromophenyl)propanoate 35 (1.01 g, $4.11 \mathrm{mmol}$ ) and 4-butylaniline 16 (0.78 mL, $4.93 \mathrm{mmol})$ were added to $\mathrm{Pd}(\mathrm{OAc}) 2(0.02 \mathrm{~g}, 0.08 \mathrm{mmol})$, BINAP $(0.10 \mathrm{~g}, 0.16 \mathrm{mmol})$ and $\mathrm{Cs}_{2} \mathrm{CO}_{3}(1.87 \mathrm{~g}, 5.75 \mathrm{mmol})$ and were allowed to react according to the general BuchwaldHartwig amination procedure. The crude residue was purified by flash column chromatography on silica eluting with hexane/ethyl acetate $(80: 20)\left(R_{f}=0.37\right)$ to afford $1.61 \mathrm{~g}$ (91\%) of 34 as a waxy yellow solid; M.p. $48-50{ }^{\circ} \mathrm{C} ;{ }^{1} \mathrm{H}$ NMR $\left(400 \mathrm{MHz} / \mathrm{CDCl}_{3}\right) / \mathrm{ppm}, \delta=0.93$ (t, $3 \mathrm{H}, J=8 \mathrm{~Hz},-\mathrm{CH}_{3} \mathrm{CH}_{2} \mathrm{CH}_{2} \mathrm{CH}_{2} \mathrm{Ar}$ ), 1.36 (sex., $2 \mathrm{H}, J=8 \mathrm{~Hz},-\mathrm{CH}_{3} \mathrm{CH}_{2} \mathrm{CH}_{2} \mathrm{CH}_{2} \mathrm{Ar}$ ), 1.57 (quin, $2 \mathrm{H}, J=8 \mathrm{~Hz},-\mathrm{CH}_{3} \mathrm{CH}_{2} \mathrm{CH}_{2} \mathrm{CH}_{2} \mathrm{Ar}$ ), 2.55 (t, $2 \mathrm{H}, J=8 \mathrm{~Hz},-\mathrm{CH}_{3} \mathrm{CH}_{2} \mathrm{CH}_{2} \mathrm{CH}_{2} \mathrm{Ar}$ ), 2.61 (t, $\left.2 \mathrm{H}, \mathrm{Ar}-\mathrm{CH}_{2} \mathrm{CH}_{2}-\left(\mathrm{COOCH}_{3}\right)\right), 2.89$ (t, $\left.2 \mathrm{H}, \mathrm{Ar}-\mathrm{CH}_{2} \mathrm{CH}_{2}\left(\mathrm{COOCH}_{3}\right)\right), 3.67$ (s, 3H, $\left.-\mathrm{COOCH}_{3}\right)$, $5.56(\mathrm{~s}, 1 \mathrm{H},-\mathrm{NH}), 6.97\left(\mathrm{~m}, 4 \mathrm{H}, \mathrm{HN}-\mathrm{ArCH}_{4}-\mathrm{CH}_{2}\left(\mathrm{COOCH}_{2} \mathrm{CH}_{3}\right)\right), 7.07$ (app. d, 2H, app. J = 8 
$\left.\mathrm{Hz}, \mathrm{C}_{4} \mathrm{H}_{9}-\mathrm{ArCH}_{4}\right) ;{ }^{13} \mathrm{C} \mathrm{NMR}\left(100 \mathrm{MHz} / \mathrm{CDCl}_{3}\right) / \mathrm{ppm}, \delta=14.0,22.4,30.2,33.9,34.9,36.0$, $51.6,117.5,118.2,129.1,129.2,132.5,135.7,140.8,142.0,173.5$; Found $[M+H]^{+}$ $\left(\mathrm{C}_{20} \mathrm{H}_{26} \mathrm{NO}_{2}\right) \mathrm{m} / \mathrm{z}=312.1958$ (Calc. 312.1964); IR (ATR) $v / \mathrm{cm}^{-1}: 3384,2960,1726,1514$, $1191,823$.

\subsubsection{Preparation of 3-(3-((4-butylphenyl)amino)phenyl)propanoic acid (36)}

Aqueous potassium hydroxide (11 mL, $2.24 \mathrm{mmol})$ was added to $30(0.35 \mathrm{~g}, 1.12 \mathrm{mmol})$ in ethanol $(6 \mathrm{~mL})$ at room temperature and allowed to react according to the general ester hydrolysis procedure to yield $0.25 \mathrm{~g}(70 \%)$ of 36 as a waxy red solid; M.p. $45-47^{\circ} \mathrm{C} ;{ }^{1} \mathrm{H}$ NMR (400 MHz/CDCl 3 )/ppm, $\delta=0.93\left(\mathrm{t}, 3 \mathrm{H}, J=8 \mathrm{~Hz},-\mathrm{CH}_{3} \mathrm{CH}_{2} \mathrm{CH}_{2} \mathrm{CH}_{2} \mathrm{Ar}\right.$ ), 1.38 (sex., $2 \mathrm{H}, J=8$ $\mathrm{Hz},-\mathrm{CH}_{3} \mathrm{CH}_{2} \mathrm{CH}_{2} \mathrm{CH}_{2} \mathrm{Ar}$ ), 1.58 (quin., $2 \mathrm{H}, J=8 \mathrm{~Hz},-\mathrm{CH}_{3} \mathrm{CH}_{2} \mathrm{CH}_{2} \mathrm{CH}_{2} \mathrm{Ar}$ ), 2.56 (t, $2 \mathrm{H}, J=8$ $\mathrm{Hz},-\mathrm{CH}_{3} \mathrm{CH}_{2} \mathrm{CH}_{2} \mathrm{CH}_{2} \mathrm{Ar}$ ), 2.67 (t, $\left.2 \mathrm{H}, J=8 \mathrm{~Hz}, \mathrm{ArC}-\mathrm{CH}_{2} \mathrm{CH}_{2}-\mathrm{COOCH}_{3}\right), 2.89$ (t, $2 \mathrm{H}, J=8 \mathrm{~Hz}$, ArC- $\mathrm{CH}_{2} \mathrm{CH}_{2}-\mathrm{COOCH}_{3}$ ), 6.72 (app. d, $\left.1 \mathrm{H}, \mathrm{HN}-\mathrm{CH}-\mathrm{C}\left(\mathrm{CH}_{2} \mathrm{CH}_{2}(\mathrm{COOH})\right)-\mathrm{CHCHCH}\right), 6.86$ (m, $2 \mathrm{H}, \mathrm{HN}-\mathrm{CH}-\mathrm{C}\left(\mathrm{CH}_{2} \mathrm{CH}_{2}(\mathrm{COOH})\right)-\mathrm{CHCHCH}$ ), 7.00 (app. d, $2 \mathrm{H}$, app. $J=8 \mathrm{~Hz}, \mathrm{HN}-\mathrm{ArCHCH}$ ), 7.09 (app. d, $1 \mathrm{H}$, app. $J=8 \mathrm{~Hz}, \mathrm{C}_{4} \mathrm{H}_{9}-\mathrm{ArCHCH}$ ), 7.16 (app. t, $2 \mathrm{H}, J=8 \mathrm{~Hz}$, $\left.\mathrm{HN}-\mathrm{CH}-\mathrm{C}\left(\mathrm{CH}_{2} \mathrm{CH}_{2}(\mathrm{COOH})\right)-\mathrm{CHCHCH}\right) ;{ }^{13} \mathrm{C} \mathrm{NMR}\left(100 \mathrm{MHz} / \mathrm{CDCl}_{3}\right) / \mathrm{ppm}, \delta=14.0,22.4$, $30.6,33.8,34.9,35.5,114.8,116.6,118.9,120.1,129.2,129.5,136.2,140.3,141.4,144.1$, 178.8; Found $[\mathrm{M}+\mathrm{Na}]^{+}\left(\mathrm{C}_{19} \mathrm{H}_{23} \mathrm{NO}_{2} \mathrm{Na}\right) \mathrm{m} / \mathrm{z}=320.1622$ (Calc. 320.1626); IR (ATR) $v / \mathrm{cm}^{-1}$ : 3407, 3102-2601 (br), 2960, 1687, 1605, 1519, 1303, 792.

\subsubsection{Preparation of 2-(4-((4-butylphenyl)amino)phenyl)acetic acid (37)}

Aqueous potassium hydroxide $(41 \mathrm{~mL}, 8.16 \mathrm{mmol})$ was added to $32(1.27 \mathrm{~g}, 4.08 \mathrm{mmol})$ in ethanol $(20 \mathrm{~mL})$ at room temperature and allowed to react according to the general ester hydrolysis procedure to yield $0.73 \mathrm{~g}(63 \%)$ of 37 as a pale orange solid; M.p. $105-108{ }^{\circ} \mathrm{C} ;{ }^{1} \mathrm{H}$ $\operatorname{NMR}\left(400 \mathrm{MHz} / \mathrm{CDCl}_{3}\right) / \mathrm{ppm}, \delta=0.93$ (t, 3H, $J=8 \mathrm{~Hz},-\mathrm{CH}_{3} \mathrm{CH}_{2} \mathrm{CH}_{2} \mathrm{CH}_{2} \mathrm{Ar}$ ), 1.38 (sex., $2 \mathrm{H}$, $J=8 \mathrm{~Hz},-\mathrm{CH}_{3} \mathrm{CH}_{2} \mathrm{CH}_{2} \mathrm{CH}_{2} \mathrm{Ar}$ ), 1.58 (quin, $2 \mathrm{H}, J=8 \mathrm{~Hz},-\mathrm{CH}_{3} \mathrm{CH}_{2} \mathrm{CH}_{2} \mathrm{CH}_{2} \mathrm{Ar}$ ), 2.56 (t, 2H, $J=$ 
$\left.8 \mathrm{~Hz}, \quad-\mathrm{CH}_{3} \mathrm{CH}_{2} \mathrm{CH}_{2} \mathrm{CH}_{2} \mathrm{Ar}\right), \quad 3.58$ (s, $\left.2 \mathrm{H}, \quad \operatorname{ArC}-\mathrm{CH}_{2}-\mathrm{COOH}\right), \quad 6.99 \quad$ (m, 4H, $\left.\mathrm{HN}-\mathrm{ArCH}_{4}-\mathrm{CH}_{2}(\mathrm{COOCH})\right), 7.08$ (app. d, 2H, app. $\mathrm{J}=8 \mathrm{~Hz}, \mathrm{HN}-\mathrm{ArCHCH}$ ), 7.14 (app. d, 2H, app. $\left.J=8 \mathrm{~Hz}, \mathrm{C}_{4} \mathrm{H}_{9}-\mathrm{ArCHCH}\right) ;{ }^{13} \mathrm{C} \mathrm{NMR}\left(100 \mathrm{MHz} / \mathrm{CDCl}_{3}\right) / \mathrm{ppm}, \delta=14.0,22.4,33.8,34.9$, $40.2,117.0,118.8,124.8,129.2,130.3,136.2,140.3,143.2,177.4$; Found $[\mathrm{M}+\mathrm{H}]^{+}$ $\left(\mathrm{C}_{18} \mathrm{H}_{22} \mathrm{NO}_{2}\right) \mathrm{m} / \mathrm{z}=284.1645$ (Calc. 284.1651); IR (ATR) v/cm ${ }^{-1}: 3374,3088-2535$ (br), 2923, 1696, 1611, 1515, 1177, 803.

\subsubsection{Preparation of 3-(4-((4-butylphenyl)amino)phenyl)propanoic acid (38)}

Aqueous potassium hydroxide $(32 \mathrm{~mL}, 6.42 \mathrm{mmol})$ was added to 34 (1.00 g, $3.21 \mathrm{mmol})$ in ethanol $(16 \mathrm{~mL})$ at room temperature and allowed to react according to the general ester hydrolysis procedure to yield $0.91 \mathrm{~g}(95 \%)$ of 38 as a pale orange solid; M.p. $118-120{ }^{\circ} \mathrm{C} ;{ }^{1} \mathrm{H}$ $\operatorname{NMR}\left(400 \mathrm{MHz} / \mathrm{CDCl}_{3}\right) / \mathrm{ppm}, \delta=0.93\left(\mathrm{t}, 3 \mathrm{H}, \mathrm{J}=8 \mathrm{~Hz},-\mathrm{CH}_{3} \mathrm{CH}_{2} \mathrm{CH}_{2} \mathrm{CH}_{2} \mathrm{Ar}\right.$ ), 1.39 (sex., 2H, $J=8 \mathrm{~Hz},-\mathrm{CH}_{3} \mathrm{CH}_{2} \mathrm{CH}_{2} \mathrm{CH}_{2} \mathrm{Ar}$ ), 1.58 (quin, $2 \mathrm{H}, \mathrm{J}=8 \mathrm{~Hz},-\mathrm{CH}_{3} \mathrm{CH}_{2} \mathrm{CH}_{2} \mathrm{CH}_{2} \mathrm{Ar}$ ), 2.55 (t, 2H, $J=$ $\left.8 \mathrm{~Hz},-\mathrm{CH}_{3} \mathrm{CH}_{2} \mathrm{CH}_{2} \mathrm{CH}_{2} \mathrm{Ar}\right), 2.66$ (t, $2 \mathrm{H}, \mathrm{Ar}-\mathrm{CH}_{2} \mathrm{CH}_{2}-\left(\mathrm{COOCH}_{3}\right)$ ), 2.90 (t, 2H, $\mathrm{Ar}-\mathrm{CH}_{2} \mathrm{CH}_{2}$ $\left.\left(\mathrm{COOCH}_{3}\right)\right), 6.97\left(\mathrm{~m}, 4 \mathrm{H}, \mathrm{HN}-\mathrm{ArCH}_{4}-\mathrm{CH}_{2}\left(\mathrm{COOCH}_{2} \mathrm{CH}_{3}\right)\right), 7.08\left(\mathrm{~m}, 4 \mathrm{H}, \mathrm{C}_{4} \mathrm{H}_{9}-\mathrm{ArCH}\right)_{4}$; ${ }^{13} \mathrm{C}$ NMR $\left(100 \mathrm{MHz} / \mathrm{CDCl}_{3}\right) / \mathrm{ppm}, \delta=14.0,22.4,30.0,33.8,34.9,35.8,117.4,118.3,129.1$, 129.2, 132.1, 135.8, 140.7, 142.2, 178.5; Found $[\mathrm{M}+\mathrm{Na}]^{+}\left(\mathrm{C}_{19} \mathrm{H}_{23} \mathrm{NO}_{2} \mathrm{Na}\right) \mathrm{m} / \mathrm{z}=320.1622$ (Calc. 320.1626); IR (ATR) v/cm-1: 3402, 3090-2607 (br), 2920, 1689, 1514, 1305, 818.

4.2.26. Preparation of mixed amine-phenol (39)

The mono-phenolic linker $5 \quad(0.40 \quad \mathrm{~g}, \quad 0.79 \quad \mathrm{mmol}), \quad 3-(3-((4-)$ butylphenyl)amino)phenyl)propanoic acid 36 (0.35 g, $1.18 \mathrm{mmol})$, DPTS (60\%) and DCC $(0.24 \mathrm{~g}, 1.18 \mathrm{mmol})$ were allowed to react according to the general mixed amine-phenol procedure. The crude product was purified by flash column chromatography on silica eluting with hexane/ethyl acetate $(80: 20)\left(R_{f}=0.39\right)$ to afford $0.52 \mathrm{~g}(84 \%)$ of 39 as a dark orange oil; ${ }^{1} \mathrm{H}$ NMR (400 MHz/CDCl 3 )/ppm, $\delta=0.87\left(\mathrm{~m}, 6 \mathrm{H},-\mathrm{CH}_{2} \mathrm{CH}_{3}\right), 0.93(\mathrm{t}, 3 \mathrm{H}, \mathrm{J}=8 \mathrm{~Hz}$, - 
$\left.\mathrm{CH}_{3} \mathrm{CH}_{2} \mathrm{CH}_{2} \mathrm{CH}_{2} \mathrm{Ar}\right), 1.16\left(\mathrm{~s}, 3 \mathrm{H},-\mathrm{CH}_{3}\right), 1.26-1.35\left(\mathrm{~m}, 10 \mathrm{H},-\mathrm{CH}_{2} \mathrm{CH}_{3}\right), 1.42\left(\mathrm{~s}, 18 \mathrm{H},-\mathrm{CH}_{3}\right.$ tertbutyl), $1.58\left(\mathrm{~m}, 3 \mathrm{H},-\mathrm{CH}_{3} \mathrm{CH}_{2} \mathrm{CH}_{2} \mathrm{CH}_{2} \mathrm{Ar}\right.$ and $\left.-\mathrm{CH}\left(\mathrm{CH}_{2} \mathrm{CH}_{3}\right)\right), 2.59\left(\mathrm{~m}, 6 \mathrm{H}, \mathrm{Ph}-\mathrm{CH}_{2} \mathrm{CH}_{2}-\mathrm{COO}-\right.$ , $-\mathrm{C}_{3} \mathrm{H}_{7} \mathrm{CH}_{2} \mathrm{Ar}, \mathrm{ArC}-\mathrm{CH}_{2} \mathrm{CH}_{2}-\mathrm{COO}$ ), 2.85 (m, 4H, Ph- $\mathrm{CH}_{2} \mathrm{CH}_{2}-\mathrm{COO}-, \mathrm{ArC}-\mathrm{CH}_{2} \mathrm{CH}_{2}-\mathrm{COO}$ ), $4.03\left(\mathrm{~m}, 2 \mathrm{H}, \mathrm{COO}-\mathrm{CH}_{2}-\mathrm{CH}\left(\mathrm{CH}_{2} \mathrm{CH}_{3}\right)-\mathrm{C}_{4} \mathrm{H}_{9}\right), 4.21\left(\mathrm{~s}, 4 \mathrm{H}, \mathrm{COO}-\mathrm{CH}_{2}-\mathrm{C}\right), 5.07(\mathrm{~s}, 1 \mathrm{H},-\mathrm{OH})$, 5.69 (s, $1 \mathrm{H},-\mathrm{NH}), 6.69$ (app. d, $1 \mathrm{H}, \mathrm{HN}-\mathrm{ArCH}^{2}-\mathrm{C}\left(\mathrm{CH}_{2} \mathrm{CH}_{3} \mathrm{COO}-\right)-\mathrm{CH}^{4} \mathrm{CH}^{5} \mathrm{CH}^{6}$ ), 6.82 (app. s, $\left.1 \mathrm{H}, \quad \mathrm{HN}-\mathrm{ArCH}^{2}-\mathrm{C}\left(\mathrm{CH}_{2} \mathrm{CH}_{3} \mathrm{COO}-\right)\right), \quad 6.87$ (app. d, $1 \mathrm{H}, \quad \mathrm{HN}-\mathrm{ArCH}^{2}-\mathrm{C}\left(\mathrm{CH}_{2} \mathrm{CH}_{3} \mathrm{COO}-\right)-$ $\mathrm{CH}^{4} \mathrm{CH}^{5} \mathrm{CH}^{6}$ ), 6.97 (s, 2H, Ph-CH), 7.00 (app. d, 2H, ArCHCHC-NH), 7.08 (app. d, 2H, $\mathrm{C}_{4} \mathrm{H}_{9}-$ $\mathrm{ArCHCH}$ ), 7.14 (app. t, $\left.1 \mathrm{H}, \mathrm{HN}-\mathrm{ArCH}^{2}-\mathrm{C}\left(\mathrm{CH}_{2} \mathrm{CH}_{3} \mathrm{COO}-\right)-\mathrm{CH}^{4} \mathrm{CH}^{5} \mathrm{CH}^{6}\right) ;{ }^{13} \mathrm{C} \mathrm{NMR}$ (100 $\left.\mathrm{MHz} / \mathrm{CDCl}_{3}\right) / \mathrm{ppm}, \delta=11.0,14.0,17.8,22.4,22.9,23.7,28.9,30.2,30.3,30.8,33.8,34.3$, 34.9, 36.2, 38.7, 46.4, 65.4, 65.6, 67.4, 114.6, 116.7, 118.7, 124.7, 126.1, 129.2, 129.4, $130.8,135.9,136.0,140.4,141.5,144.1,152.2,172.4,172.7,172.9$; Found $[\mathrm{M}+\mathrm{H}]^{+}$ $\left(\mathrm{C}_{49} \mathrm{H}_{72} \mathrm{NO}_{7}\right) \mathrm{m} / \mathrm{z}=786.5303$ (Calc. 786.5309 ); IR (ATR) $v / \mathrm{cm}^{-1}: 3634,3380,2960,1731$, $1515,1135,752$.

\subsubsection{Preparation of mixed amine-phenol (40)}

The mono-phenolic linker 5 (0.51 g, $1.01 \mathrm{mmol})$, 2-(4-((4-butylphenyl)amino)phenyl)acetic acid $37(0.43 \mathrm{~g}, 1.52 \mathrm{mmol})$, DPTS (60\%) and DCC (0.31 g, $1.52 \mathrm{mmol})$ were allowed to react according to the general mixed amine-phenol procedure. The crude product was purified by flash column chromatography on silica eluting with hexane/ethyl acetate (85:15) $\left(\mathrm{R}_{\mathrm{f}}=0.33\right)$ to afford $0.42 \mathrm{~g}(54 \%)$ of 40 as an orange oil; ${ }^{1} \mathrm{H} \mathrm{NMR}\left(400 \mathrm{MHz} / \mathrm{CDCl}_{3}\right) / \mathrm{ppm}, \delta=$ $0.87\left(\mathrm{~m}, 6 \mathrm{H},-\mathrm{CH}_{2} \mathrm{CH}_{3}\right), 0.93\left(\mathrm{t}, 3 \mathrm{H}, J=8 \mathrm{~Hz},-\mathrm{CH}_{3} \mathrm{CH}_{2} \mathrm{CH}_{2} \mathrm{CH}_{2} \mathrm{Ar}\right), 1.16\left(\mathrm{~s}, 3 \mathrm{H},-\mathrm{CH}_{3}\right), 1.26-$ $1.36\left(\mathrm{~m}, 10 \mathrm{H},-\mathrm{CH}_{2} \mathrm{CH}_{3}\right), 1.42$ (s, $18 \mathrm{H},-\mathrm{CH}_{3}$ tert-butyl), 1.57 (m, 3H, $-\mathrm{CH}_{3} \mathrm{CH}_{2} \mathrm{CH}_{2} \mathrm{CH}_{2} \mathrm{Ar}$ and $\left.-\mathrm{CH}\left(\mathrm{CH}_{2} \mathrm{CH}_{3}\right)\right), 2.56\left(\mathrm{~m}, 4 \mathrm{H}, \mathrm{Ph}-\mathrm{CH}_{2} \mathrm{CH}_{2}-\mathrm{COO}-\right.$ and $\left.-\mathrm{C}_{3} \mathrm{H}_{7} \mathrm{CH}_{2} \mathrm{Ar}\right), 2.82(\mathrm{t}, 2 \mathrm{H}, J=8 \mathrm{~Hz}, \mathrm{Ph}-$ $\mathrm{CH}_{2} \mathrm{CH}_{2}-\mathrm{COO}$ ), 3.53 (s, 2H, ArC- $\left.\mathrm{CH}_{2}-\mathrm{COO}\right), 4.00$ (m, 2H, $\left.\mathrm{COO}-\mathrm{CH}_{2}-\mathrm{CH}_{(}\left(\mathrm{CH}_{2} \mathrm{CH}_{3}\right)-\mathrm{C}_{4} \mathrm{H}_{9}\right)$, $4.21\left(\mathrm{~m}, 4 \mathrm{H}, \mathrm{COO}-\mathrm{CH}_{2}-\mathrm{C}\right), 5.07(\mathrm{~s}, 1 \mathrm{H},-\mathrm{OH}), 5.60(\mathrm{~s}, 1 \mathrm{H},-\mathrm{NH}), 6.97(\mathrm{~m}, 6 \mathrm{H}, \mathrm{Ph}-\mathrm{CH}$ and $\mathrm{ArCH}-\mathrm{CNH}), 7.10\left(\mathrm{~m}, 4 \mathrm{H}, \mathrm{ArCH}-\mathrm{CCH}_{2}\right) ;{ }^{13} \mathrm{C} \mathrm{NMR}\left(100 \mathrm{MHz} / \mathrm{CDCl}_{3}\right) / \mathrm{ppm}, \delta=11.0,14.0$, 
$14.1,17.8,22.4,22.9,23.7,28.9,30.3,30.8,33.8,34.3,34.9,36.2,38.7,40.4,46.4,65.4$, $65.8,67.4,117.0,118.6,124.7,125.3,129.2,130.1,130.8,135.9,136.1,140.4,143.0,152.2$, 171.4, 172.6, 172.8; Found $[\mathrm{M}+\mathrm{H}]^{+}\left(\mathrm{C}_{48} \mathrm{H}_{70} \mathrm{NO}_{7}\right) \mathrm{m} / \mathrm{z}=772.5147$ (Calc. 771.5152$)$; IR (ATR) $v / \mathrm{cm}^{-1}: 3632,3382,2960,1730,1514,1132,753$.

\subsubsection{Preparation of mixed amine-phenol (41)}

The mono-phenolic linker $5 \quad(0.46 \quad \mathrm{~g}, \quad 0.90 \quad \mathrm{mmol}), \quad 3-(4-(4-$ butylphenyl)amino)phenyl)propanoic acid $38(0.40 \mathrm{~g}, 1.34 \mathrm{mmol})$, DPTS (60\%) and DCC $(0.28 \mathrm{~g}, 1.34 \mathrm{mmol})$ were allowed to react according to the general mixed amine-phenol procedure. The crude product was purified by flash column chromatography on silica eluting with hexane/ethyl acetate $(80: 20)\left(R_{f}=0.37\right)$ to afford $0.64 \mathrm{~g}(90 \%)$ of 41 as a purple oil; ${ }^{1} \mathrm{H}$ NMR $\left(400 \mathrm{MHz} / \mathrm{CDCl}_{3}\right) / \mathrm{ppm}, \delta=0.87\left(\mathrm{~m}, 6 \mathrm{H},-\mathrm{CH}_{2} \mathrm{CH}_{3}\right), 0.93(\mathrm{t}, 3 \mathrm{H}, J=8 \mathrm{~Hz},-$ $\left.\mathrm{CH}_{3} \mathrm{CH}_{2} \mathrm{CH}_{2} \mathrm{CH}_{2} \mathrm{Ar}\right), 1.16\left(\mathrm{~s}, 3 \mathrm{H},-\mathrm{CH}_{3}\right), 1.27-1.36\left(\mathrm{~m}, 10 \mathrm{H},-\mathrm{CH}_{2} \mathrm{CH}_{3}\right), 1.42\left(\mathrm{~s}, 18 \mathrm{H},-\mathrm{CH}_{3}\right.$ tertbutyl), $1.57\left(\mathrm{~m}, 3 \mathrm{H},-\mathrm{CH}_{3} \mathrm{CH}_{2} \mathrm{CH}_{2} \mathrm{CH}_{2} \mathrm{Ar}\right.$ and $\left.-\mathrm{CH}\left(\mathrm{CH}_{2} \mathrm{CH}_{3}\right)\right), 2.59\left(\mathrm{~m}, 6 \mathrm{H}, \mathrm{Ph}-\mathrm{CH}_{2} \mathrm{CH}_{2}-\mathrm{COO}\right.$ , $-\mathrm{C}_{3} \mathrm{H}_{7} \mathrm{CH}_{2} \mathrm{Ar}, \mathrm{ArC}-\mathrm{CH}_{2} \mathrm{CH}_{2}-\mathrm{COO}$ ), 2.85 (m, 4H, Ph- $\mathrm{CH}_{2} \mathrm{CH}_{2}-\mathrm{COO}-, \mathrm{ArC}-\mathrm{CH}_{2} \mathrm{CH}_{2}-\mathrm{COO}$ ), $4.03\left(\mathrm{~m}, 2 \mathrm{H}, \mathrm{COO}-\mathrm{CH}_{2}-\mathrm{CH}\left(\mathrm{CH}_{2} \mathrm{CH}_{3}\right)-\mathrm{C}_{4} \mathrm{H}_{9}\right), 4.21\left(\mathrm{~s}, 4 \mathrm{H}, \mathrm{COO}-\mathrm{CH}_{2}-\mathrm{C}\right), 5.07(\mathrm{~s}, 1 \mathrm{H},-\mathrm{OH})$, $5.56(\mathrm{~s}, 1 \mathrm{H},-\mathrm{NH}), 6.96(\mathrm{~m}, 6 \mathrm{H}, \mathrm{Ph}-\mathrm{CH}$ and $\mathrm{ArCH}-\mathrm{CNH}), 7.06\left(\mathrm{~m}, 4 \mathrm{H}, \mathrm{ArCH}-\mathrm{CCH}_{2}\right) ;{ }^{13} \mathrm{C} \mathrm{NMR}$ $\left(100 \mathrm{MHz} / \mathrm{CDCl}_{3}\right) / \mathrm{ppm}, \delta=11.0,14.0,17.8,22.4,22.9,23.7,28.9,30.3,30.8,33.8,34.3$, 34.9, 35.9, 36.2, 38.7, 46.4, 65.5, 67.3, 117.5, 118.2, 124.7, 126.1, 129.0, 129.2, 130.8, 132.2, 135.7, 136.0, 140.8, 142.1, 152.2, 172.5, 172.7, 172.9; Found $[\mathrm{M}+\mathrm{H}]^{+}\left(\mathrm{C}_{49} \mathrm{H}_{72} \mathrm{NO}_{7}\right) \mathrm{m} / \mathrm{z}$ $=786.5303$ (Calc. 786.5309); IR (ATR) v/cm-1:3633, 3384, 2960, 1731, 1514, 1136, 753.

4.2.29. Preparation of bis(diphenylamine) (42)

The first generation hydroxyl linker $6 \begin{array}{llllll}0.08 & \mathrm{~g}, & 0.35 & \mathrm{mmol}), & 3-(3-((4-)\end{array}$ butylphenyl)amino)phenyl)propanoic acid $36(0.24 \mathrm{~g}, 0.81 \mathrm{mmol})$, DPTS $(60 \%)$ and DCC $(0.17 \mathrm{~g}, 0.81 \mathrm{mmol})$ were allowed to react according to the general bis(diphenylamine) 
procedure. The crude product was purified by flash column chromatography on silica eluting with hexane/ethyl acetate $(80: 20)\left(R_{f}=0.38\right)$ to afford $0.21 \mathrm{~g}(75 \%)$ of 42 as a pale orange oil; ${ }^{1} \mathrm{H}$ NMR $\left(400 \mathrm{MHz} / \mathrm{CDCl}_{3}\right) / \mathrm{ppm}, \delta=0.86\left(\mathrm{~m}, 6 \mathrm{H},-\mathrm{CH}_{2} \mathrm{CH}_{3}\right), 0.93(\mathrm{t}, 6 \mathrm{H}, J=8$ $\left.\mathrm{Hz},-\mathrm{CH}_{3} \mathrm{CH}_{2} \mathrm{CH}_{2} \mathrm{CH}_{2} \mathrm{Ar}\right), 1.15\left(\mathrm{~s}, 3 \mathrm{H},-\mathrm{CH}_{3}\right), 1.26-1.39\left(\mathrm{~m}, 12 \mathrm{H},-\mathrm{CH}_{2} \mathrm{CH}_{3}\right), 1.58(\mathrm{~m}$, $5 \mathrm{H},-\mathrm{CH}_{3} \mathrm{CH}_{2} \mathrm{CH}_{2} \mathrm{CH}_{2} \mathrm{Ar}$ and $\left.-\mathrm{CH}\left(\mathrm{CH}_{2} \mathrm{CH}_{3}\right)\right), 2.55\left(\mathrm{t}, 4 \mathrm{H}, J=8 \mathrm{~Hz},-\mathrm{C}_{3} \mathrm{H}_{7} \mathrm{CH}_{2} \mathrm{Ar}\right.$ ), 2.60 (t, $4 \mathrm{H}$, $J=8 \mathrm{~Hz}, \mathrm{ArC}-\mathrm{CH}_{2} \mathrm{CH}_{2}-\mathrm{COO}$ ), $2.86\left(\mathrm{t}, 4 \mathrm{H}, J=8 \mathrm{~Hz}, \mathrm{ArC}-\mathrm{CH}_{2} \mathrm{CH}_{2} \mathrm{COO}\right), 4.02(\mathrm{~m}, 2 \mathrm{H}$, $\left.\mathrm{COO}-\mathrm{CH}_{2}-\mathrm{CH}\left(\mathrm{CH}_{2} \mathrm{CH}_{3}\right)-\mathrm{C}_{4} \mathrm{H}_{9}\right), 4.19\left(\mathrm{~m}, 4 \mathrm{H}, \mathrm{COO}-\mathrm{CH}_{2}-\mathrm{C}\right), 5.67$ (s, 2H, -NH), 6.68 (app. d, $\left.2 \mathrm{H}, \quad J=8 \mathrm{~Hz}, \mathrm{HN}-\mathrm{ArCH}^{2}-\mathrm{C}\left(\mathrm{CH}_{2} \mathrm{CH}_{3} \mathrm{COO}-\right)-\mathrm{CH}^{4} \mathrm{CH}^{5} \mathrm{CH}^{6}-\right), 6.80$ (m, $2 \mathrm{H}, \mathrm{HN}-\mathrm{ArCH}^{2-}$ $\left.\mathrm{C}\left(\mathrm{CH}_{2} \mathrm{CH}_{3} \mathrm{COO}-\right)\right), 6.87$ (m, 2H, HN-ArCH${ }^{2} \mathrm{C}\left(\mathrm{CH}_{2} \mathrm{CH}_{3} \mathrm{COO}^{-}\right)-\mathrm{CH}^{4} \mathrm{CH}^{5} \mathrm{CH}^{6}$ ), 6.99 (app. d, 4H, app. $J=8 \mathrm{~Hz}, \mathrm{ArCHCHC}-\mathrm{NH}$ ), 7.10 (app. d, 4H, app. $J=8 \mathrm{~Hz}, \mathrm{C}_{4} \mathrm{H}_{9}-\mathrm{ArCHCH}$ ), 7.13 (app. t, 2H, app. $\left.\mathrm{J}=8 \mathrm{~Hz}, \mathrm{HN}-\mathrm{ArCH}^{2}-\mathrm{C}\left(\mathrm{CH}_{2} \mathrm{CH}_{3} \mathrm{COO}-\right)-\mathrm{CH}^{4} \mathrm{CH}^{5} \mathrm{CH}^{6}-\right) ;{ }^{13} \mathrm{C} \mathrm{NMR}(100$ $\left.\mathrm{MHz} / \mathrm{CDCl}_{3}\right) / \mathrm{ppm}, \delta=11.0,14.0,17.8,22.4,22.9,23.7,28.9,30.3,30.8,31.5,33.8,34.9$, $35.5,38.7,46.3,65.5,67.4,114.6,116.8,118.7,120.1,129.2,129.4,136.0,140.4,141.5$, 144.1, 172.4, 172.9; Found $\left[\mathrm{M}+\mathrm{H}^{+}\left(\mathrm{C}_{51} \mathrm{H}_{69} \mathrm{~N}_{2} \mathrm{O}_{6}\right) \mathrm{m} / \mathrm{z}=805.5155\right.$ (Calc. 805.5156); IR (ATR) $v / \mathrm{cm}^{-1}:$ 3382, 2960, 1730, 1514, 1134, 776.

\subsubsection{Preparation of bis(diphenylamine) (43)}

The first generation hydroxyl linker $6 \quad(0.19 \mathrm{~g}, \quad 0.86 \quad \mathrm{mmol}), \quad 2-(4-(4-$ butylphenyl)amino)phenyl)acetic acid 37 (0.56 g, $1.98 \mathrm{mmol})$, DPTS (60\%) and DCC (0.41 $\mathrm{g}, 1.98 \mathrm{mmol}$ ) were allowed to react according to the general bis(diphenylamine) procedure. The crude product was purified by flash column chromatography on silica eluting with hexane/ethyl acetate $(80: 20)\left(R_{f}=0.41\right)$ to afford $0.46 \mathrm{~g}(68 \%)$ of 43 as a dark red oil; ${ }^{1} \mathrm{H}$ NMR $\left(400 \mathrm{MHz} / \mathrm{CDCl}_{3}\right) / \mathrm{ppm}, \quad \delta=0.87\left(\mathrm{~m}, 6 \mathrm{H},-\mathrm{CH}_{2} \mathrm{CH}_{3}\right), 0.93(\mathrm{t}, 6 \mathrm{H}, \quad J=8$ $\left.\mathrm{Hz},-\mathrm{CH}_{3} \mathrm{CH}_{2} \mathrm{CH}_{2} \mathrm{CH}_{2} \mathrm{Ar}\right), 1.15$ (s, $\left.3 \mathrm{H},-\mathrm{CH}_{3}\right), 1.25-1.30\left(\mathrm{~m}, 8 \mathrm{H},-\mathrm{CH}_{2} \mathrm{CH}_{3}\right), 1.36(\mathrm{~m}$, $\left.4 \mathrm{H},-\mathrm{CH}_{3} \mathrm{CH}_{2} \mathrm{CH}_{2} \mathrm{CH}_{2} \mathrm{Ar}\right), 1.58\left(\mathrm{~m}, 5 \mathrm{H},-\mathrm{CH}_{3} \mathrm{CH}_{2} \mathrm{CH}_{2} \mathrm{CH}_{2} \mathrm{Ar}\right.$ and $\left.-\mathrm{CH}\left(\mathrm{CH}_{2} \mathrm{CH}_{3}\right)\right), 2.55$ (t, $4 \mathrm{H}, J$ $=8 \mathrm{~Hz},-\mathrm{CH}_{3} \mathrm{CH}_{2} \mathrm{CH}_{2} \mathrm{CH}_{2} \mathrm{Ar}$ ), $3.50\left(\mathrm{~s}, 4 \mathrm{H}, \mathrm{ArC}-\mathrm{CH}_{2}-\mathrm{COO}\right), 3.98\left(\mathrm{~m}, 2 \mathrm{H}, \mathrm{COO}-\mathrm{CH}_{2}\right.$ 
$\left.\mathrm{CH}\left(\mathrm{CH}_{2} \mathrm{CH}_{3}\right)-\mathrm{C}_{4} \mathrm{H}_{9}\right), 4.16\left(\mathrm{~d}, 2 \mathrm{H}, \mathrm{J}=12 \mathrm{~Hz}, \mathrm{COO}-\mathrm{CH}_{2} \mathrm{C}\right), 4.22\left(\mathrm{~d}, 2 \mathrm{H}, \mathrm{J}=12 \mathrm{~Hz}, \mathrm{COO}-\mathrm{CH}_{2}\right.$ C), 5.59 (s, 2H, -NH), 6.94 (app. d, 4H, $\mathrm{ArCH}-\mathrm{CNH}$ ), 6.98 (app. d, 4H, $\mathrm{ArCH}-\mathrm{CNH}$ ), 7.07 (m, $\left.\left.8 \mathrm{H}, \mathrm{ArCH}-\mathrm{CCH}_{2}\right) ;{ }^{13} \mathrm{C} \mathrm{NMR} \mathrm{(100} \mathrm{MHz/CDCl} 3\right) / p p m, \delta=10.9,14.0,17.9,22.4,23.0,28.9$, $30.3,31.5,33.8,34.9,38.6,40.4,46.3,65.6,67.4,117.1,118.6,125.3,129.2,130.1,136.0$, 140.4, 142.9, 171.4, 172.8; Found $[\mathrm{M}+\mathrm{H}]^{+}\left(\mathrm{C}_{49} \mathrm{H}_{65} \mathrm{~N}_{2} \mathrm{O}_{6}\right) \mathrm{m} / \mathrm{z}=777.4839$ (Calc. 777.4843); IR (ATR) $v / \mathrm{cm}^{-1}: 3379,2960,1730,1607,1514,1131$.

\subsubsection{Preparation of bis(diphenylamine) (44)}

The first generation hydroxyl linker $6 \begin{array}{lllll}0.13 & \mathrm{~g}, & 0.58 & \mathrm{mmol}) \text {, } & 3-\left(4-\left(4^{-}\right.\right.\end{array}$ butylphenyl)amino)phenyl)propanoic acid 38 (0.40 g, $1.34 \mathrm{mmol})$, DPTS (60\%) and DCC $(0.28 \mathrm{~g}, 1.34 \mathrm{mmol})$ were allowed to react according to the general bis(diphenylamine) procedure. The crude product was purified by flash column chromatography on silica eluting with hexane/ethyl acetate $(80: 20)\left(R_{f}=0.42\right)$ to afford $0.45 \mathrm{~g}(96 \%)$ of 44 as a pale yellow oil; ${ }^{1} \mathrm{H}$ NMR $\left(400 \mathrm{MHz} / \mathrm{CDCl}_{3}\right) / \mathrm{ppm}, \delta=0.87\left(\mathrm{~m}, 6 \mathrm{H},-\mathrm{CH}_{2} \mathrm{CH}_{3}\right), 0.92$ (t, $6 \mathrm{H}, \quad J=8$ $\left.\mathrm{Hz}, \quad-\mathrm{CH}_{3} \mathrm{CH}_{2} \mathrm{CH}_{2} \mathrm{CH}_{2} \mathrm{Ar}\right), \quad 1.17 \quad\left(\mathrm{~s}, \quad 3 \mathrm{H}, \quad-\mathrm{CH}_{3}\right), \quad 1.27-1.38 \quad\left(\mathrm{~m}, \quad 12 \mathrm{H}, \quad-\mathrm{CH}_{2} \mathrm{CH}_{3}\right.$ and $\left.-\mathrm{CH}_{3} \mathrm{CH}_{2} \mathrm{CH}_{2} \mathrm{CH}_{2} \mathrm{Ar}\right), 1.57\left(\mathrm{~m}, 8 \mathrm{H},-\mathrm{CH}_{3} \mathrm{CH}_{2} \mathrm{CH}_{2} \mathrm{CH}_{2} \mathrm{Ar}\right.$ and $\left.-\mathrm{CH}\left(\mathrm{CH}_{2} \mathrm{CH}_{3}\right)\right)$, 2.57 (m, $8 \mathrm{H}, \quad-\mathrm{CH}_{3} \mathrm{CH}_{2} \mathrm{CH}_{2} \mathrm{CH}_{2} \mathrm{Ar}$ and $\left.\mathrm{Ar}-\mathrm{CH}_{2} \mathrm{CH}_{2}-(\mathrm{COO}-)\right), 2.85$ (t, 4H, J=8 Hz, $\mathrm{Ar}-\mathrm{CH}_{2} \mathrm{CH}_{2}$ $\left.\left(\mathrm{COOCH}_{3}\right)\right), 4.03\left(\mathrm{~m}, 2 \mathrm{H}, \mathrm{COO}-\mathrm{CH}_{2}-\mathrm{CH}\left(\mathrm{CH}_{2} \mathrm{CH}_{3}\right)-\mathrm{C}_{4} \mathrm{H}_{9}\right), 4.19$ (s, 4H, COO-CH$\left.{ }_{2}-\mathrm{C}\right), 5.55$ (s, $2 \mathrm{H}, \quad-\mathrm{NH}), \quad 6.95\left(\mathrm{~m}, \quad 8 \mathrm{H}, \quad \mathrm{ArCH}-\mathrm{CCH}_{2}\right), \quad 7.05(\mathrm{~m}, \quad 8 \mathrm{H}, \quad \mathrm{ArCH}-\mathrm{CNH}) ;{ }^{13} \mathrm{C}$ NMR $\quad(100$ $\left.\mathrm{MHz} / \mathrm{CDCl}_{3}\right) / \mathrm{ppm}, \delta=11.0,14.0,17.8,22.4,22.9,23.7,28.9,30.1,30.3,33.8,34.9,35.9$, $38.7,46.3,65.5,67.4,117.5,118.2,129.0,129.2,132.2,135.7,140.8,142.0,172.5,172.9$; Found $[\mathrm{M}+\mathrm{H}]^{+}\left(\mathrm{C}_{51} \mathrm{H}_{69} \mathrm{~N}_{2} \mathrm{O}_{6}\right) \mathrm{m} / \mathrm{z}=805.5150$ (Calc. 805.5156); IR (ATR) v/cm ${ }^{-1}:$ 3384, 2960, $1730,1514,1137,752$.

\section{Acknowledgements}


The authors acknowledge the UK Biotechnology and Biological Sciences Research Council (BBSRC) and BP p.I.c. for financial support. Use of the Chemical Analysis Facility (CAF) at the University of Reading and the Analytical Department at the BP Technology Centre, Pangbourne are gratefully acknowledged.

\section{References}

[1] Santos JCO, Santos IMG., Sinfrônio FSM, Silva MA, Sobrinho EV, J. Therm. Anal. Calorim. 2005; 79:461.

[2] Dantas TNC, Dantas MSG, Dantas AAN, Ornellas CVD, Queiroz LR, Fuel 2003; 82:1465.

[3] Rios MADS, J. Therm. Anal. Calorim. 2013; 111:553.

[4] Sevim ZE, Svajus A, Ind. Crop. Prod. 2000; 11:277.

[5] Keating MY, Howell JL, J. Therm. Anal. Calorim. 2011; 106:213.

[6] Ren D, Gellman AJ, Tribol. Int. 2001; 34:353.

[7] Duangkaewmanee S, Petsom A, Tribol. Int. 2011; 44:266.

[8] Rios MADS, Mazzetto SE, J. Therm. Anal. Calorim. 2013; 111:553.

[9] Du D, Kim SS, Moon WS, Jin SB, Kwon WS, Thermochim. Acta. 2003; 407:17.

[10] He JB, Shi H, Wang Y, Gao XL, Molecules 2018; 23:401.

[11] Jing Y, Diao Y, Yu X, React. Funct. Polym. 2019; 135:16.

[12] Ingold KU, Chem. Rev. 1961; 61:563.

[13] Ingold KU, Pratt DA, Chem. Rev. 2014; 114:9022.

[14] Shah R, Haidasz EA, Valgimigli L, Pratt DA, J. Am. Chem. Soc. 2015; 137:2440.

[15] Czochara R, Kusio J, Symonowicz M, Litwinienko G, Ind. Eng. Chem. Res. 2016; 55:9887.

[16] Bolbukh Y, Kuzema P, Tertykh V, Laguta I, J. Therm. Anal. Calorim. 2008; 94:727.

[17] Gensler R, Plummer CJG, Kausch HH, Kramer E, Pauquet JR, Zweifel H, Polym. Degrad. Stab. 2000; 67:195.

[18] Miao C, Zhang L, Zheng K, Cui Y, Zhang S, Yu L, Zhang P, Tribol. Int. 2015; 88:95.

[19] Gatto VJ, Elnagar HY, Moehle WE, Schneller ER, Lubr. Sci. 2007; 19:25.

[20] Valgimigli L, Pratt DA, Acc. Chem. Res. 2015; 48:966.

[21] Hanthorn JJ, Valgimigli L, Pratt DA, J. Org. Chem. 2012; 77:6908.

[22] Feng J, Zhao H, Yue S, Liu S, ACS Sustainable Chem. Eng. 2017; 5:3399. 
[23] Duangkaewmanee S, Petsom A, Tribol. Int. 2011; 44:266.

[24] Chao TS, Hutchison DA, Kjonaas M, Ind. Eng. Chem. Prod. Res. Dev. 1984; 23:21.

[25] Fotty R, Rakovsky S, Marchevsky P, Popov AA, Oxid. Commun. 1997; 20:208.

[26] Gatto VJ, Grina MA, Lubr. Eng. 1999; 55:11.

[27] Sharma BK, Perez JM, Erhan SZ, Energy Fuels 2007; $21: 2408$.

[28] Kassler A, Pittenauer E, Doerr N, Allmaier G, Rapid Commun. Mass Spectrum. 2014; 28:63.

[29] Higgins CL, Fillip SV, Afsar A, Hayes W, React. Funct. Polym. 2019; 142:119.

[30] Kumar S, Johansson H, Kanda T, Engman L, Müller T, Jonsson M, Pedulli GF, Petrucci S, Valgimigli L, Org. Lett. 2008; 10:4895.

[31] Gatto VJ, Elnagar HY, Moehle WE, Schneller ER, lubrication Science 2007; 19:25.

[32] Hanthorn JJ, Valgimigli L, Pratt DA, J. Am. Chem. Soc. 2012; 134:8306.

[33] Valgimigli L, Brigati G, Pedulli GF, DiLabio GA, Mastragostino M, Arbizzani C, Pratt DA, Chem. Eur. J. 2003; 9:4997.

[34] Miao C, Zhang Y, Yang G, Zhang S, Yu L, Zhang P, Ind. Eng. Chem. Res. 2016; 55:12703.

[35] Li C, Sun P, Guo S, Zhang Z, Wang J, J. Appl. Polym. Sci. 2017; 134:45095.

[36] Yu S, Feng J, Cai T, Liu S, Ind. Eng. Chem. Res. 2017; 56:4196.

[37] Louie J, Hartwig JF, Tetrahedron Lett. 1995; 36:3609.

[38] Driver MS, Hartwig JF, J. Am. Chem. Soc. 1996; 118:7217.

[39] Guram AS, Rennels RA, Buchwald SL, Angew. Chemie Int. Ed. 1995; 34:1348.

[40] Suarez-Pantiga S, Hernandez-Ruiz R, Virumbrales C, Pedrosa MR, Sanz R, Angew. Chemie Int. Ed. 2019; 58:2129.

[41] Vantourout JC, Law RP, Isidro-Llobet A, Atkinson SJ, Watson AJB, J. Org. Chem. 2016; 81:3942.

[42] Park NH, Teverovskiy G, Buchwald SL, Org. Lett. 2014; 16:220.

[43] Graboyes H, Anderson EL, Levinson SH, Resnick TM, J. Heterocycl. Chem. 1975; 12:1225.

[44] Acheson RM, Bolton RG, J. Chem. Soc. Perkin 1 1975; 1975:650.

[45] Huang X, Anderson KW, Zim D, Jiang L, Klapars A, Buchwald SL, J. Am. Chem. Soc. 2003; 125:6653. 


\section{Tables, Figures and schemes captions}

Figure 1. Structure of Schiff base bridged phenolic diphenylamine (SPD) antioxidants SPD1 and SPD2 [37].

Figure 2. Commercial antioxidants a) Irganox L135 and b) L57.

Scheme 1. The statistical reaction between 3-(3,5 di-tert-butyl-4-hydroxyphenyl)propionic acid 7,4 and 6 to yield the mixed amine-phenol 8 .

Figure 3. Potential products from the statistical reaction shown in Scheme 1.

Scheme 2. Synthesis of the mono-phenolic hydroxyl linker 5 and mixed amine-phenol 8.

Table 1. Structure of the meta substituted amine-phenol 8 and bis(diphenylamine) 9 for comparison against the para substituted amine-phenol 11 and bis(diphenylamine) 13.

Scheme 3. General scheme for the synthesis of Series 2 diphenylamine derivatives.

Table 2. Structure of the ortho, meta and para substituted amine-phenols 24-26 and the bis(diphenylamines) 27-29 for comparison.

Scheme 4 General scheme for the synthesis of Series 3 diphenylamine derivatives.

Table 3. Structure of the mixed amine-phenols 39-41 and the bis(diphenylamines) 42-44 for comparison

Figure 4. Average Oxidation induction time of $0.5 \% \mathrm{w} / \mathrm{w}$ antioxidant-base oil samples (tested in duplicate).

Figure 5. Average Oxidation onset temperature of $0.5 \% \mathrm{w} / \mathrm{w}$ antioxidant-base oil samples (tested in duplicate).

Figure 6. Average Oxidation induction time of Series 2, mixed amine-phenol base oil samples (tested in duplicate).

Figure 7. Comparison of oxidation induction time analysis from Series $\mathbf{1}$ and 2.

Figure 8. Oxidation onset temperature analysis of Series 2, mixed amine-phenol base oil samples. 
Figure 9. Oxidation induction time analysis of bis(diphenylamines) 27-29.

Figure 10. Oxidation induction time analysis of Series 3, mixed amine-phenol base oil samples.

Figure 11. Comparison of average oxidation induction time analysis from Series 1 (8), Series 2 (25) and Series 3 (39).

Figure 12. Average Oxidation onset temperature analysis of Series 3, mixed amine-phenol base oil samples (tested in duplicate). 
Figures and Schemes

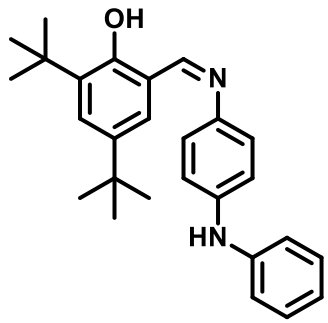

SPD1

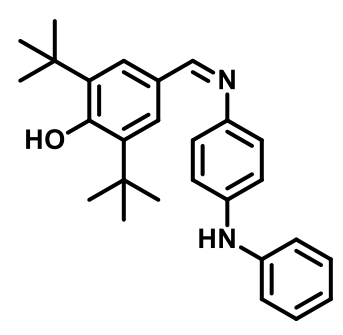

SPD2

Figure 1

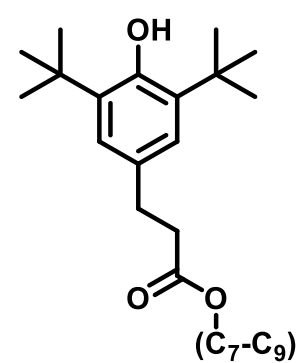

a)<smiles>[R][R]1ccc(Nc2ccc([R])cc2)cc1</smiles>

b)

Figure 2 


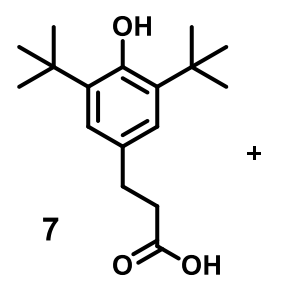

$\underbrace{\mathrm{N}}_{4} \underbrace{\mathrm{O}}_{\mathrm{HO}^{2}}$<smiles>CCCCC(CC)COC(=O)C(COCC(=O)CCc1cc(C(C)(C)C)c(O)c(C(C)(C)C)c1)(COC(=O)c1cccc(Nc2ccccc2)c1)C(=O)OC(C)(C)C</smiles>

Scheme 1
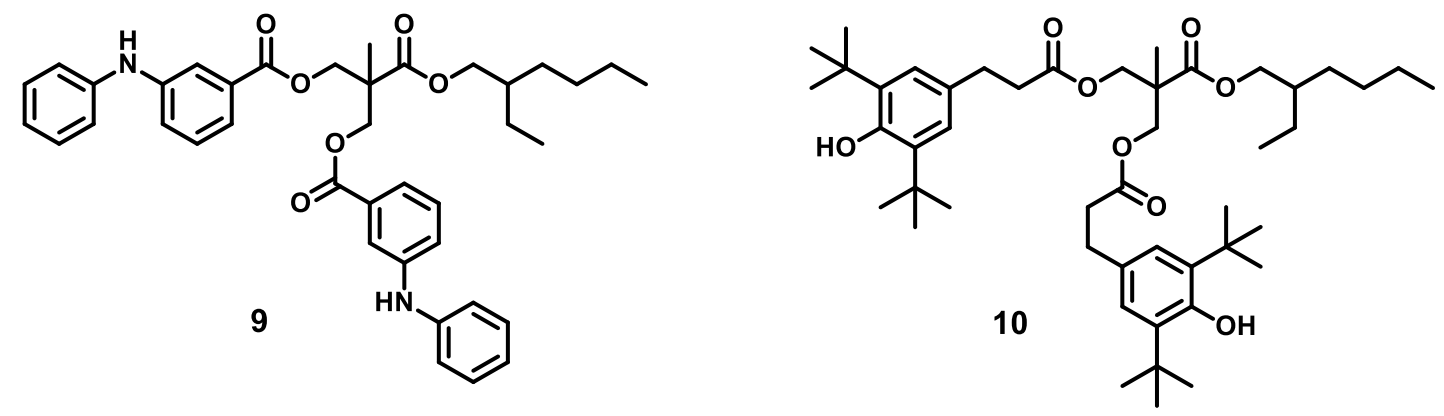

Figure 3 


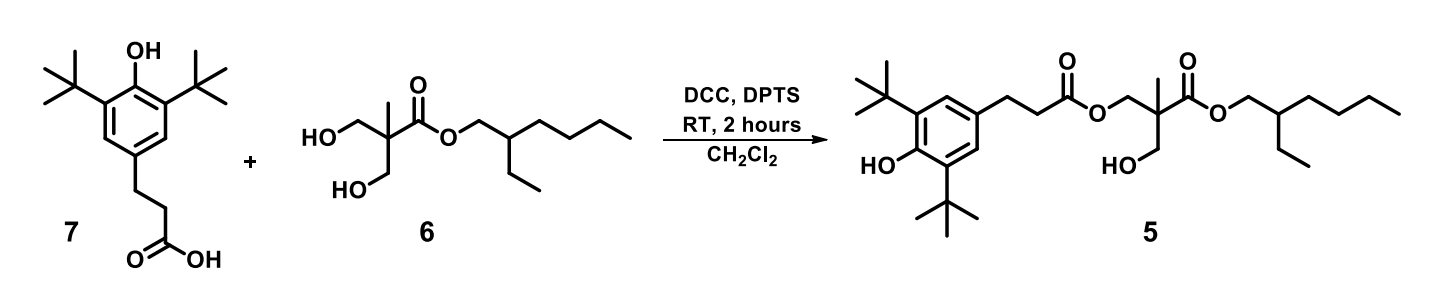

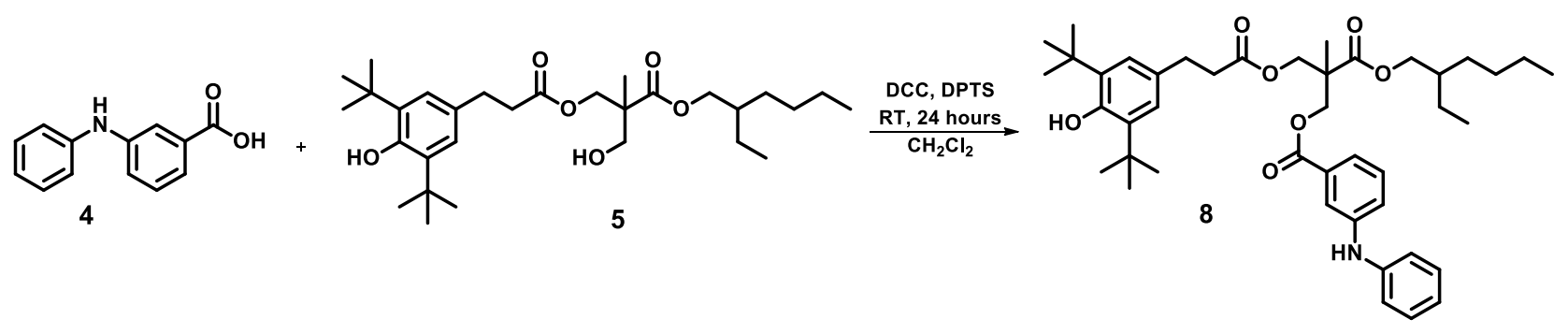

Scheme 2

Table 1

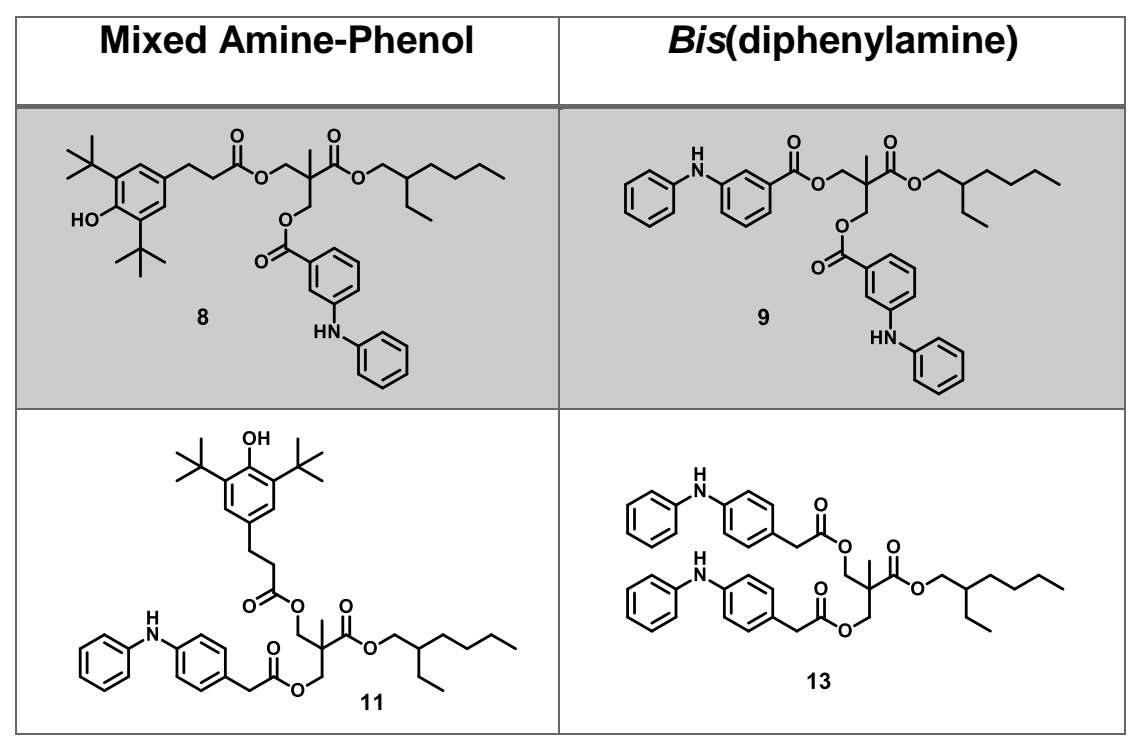



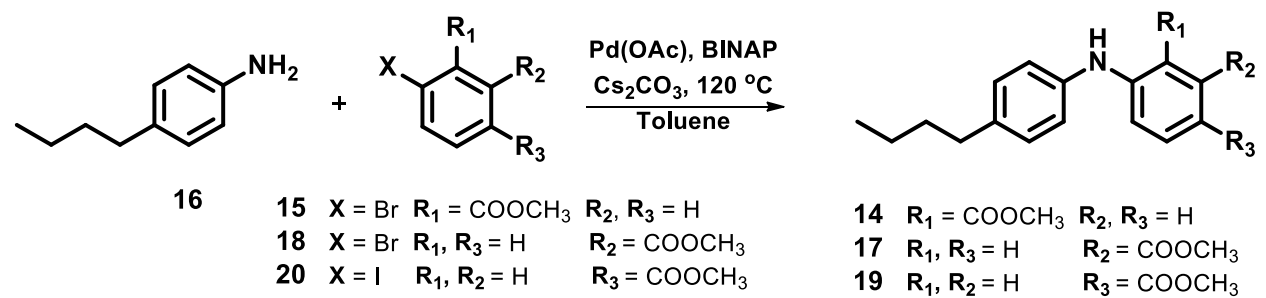

$14 \quad \mathbf{R}_{\mathbf{1}}=\mathrm{COOCH}_{3} \mathbf{R}_{\mathbf{2}}, \mathbf{R}_{\mathbf{3}}=\mathrm{H}$

$17 \quad \mathbf{R}_{\mathbf{1}}, \mathbf{R}_{\mathbf{3}}=\mathrm{H} \quad \mathbf{R}_{\mathbf{2}}=\mathrm{COOCH}_{3}$

$19 \mathbf{R}_{\mathbf{1}}, \mathbf{R}_{\mathbf{2}}=\mathrm{H} \quad \mathbf{R}_{\mathbf{3}}=\mathrm{COOCH}_{3}$

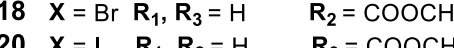

KOH EtOH/ $/ \mathrm{H}_{2} \mathrm{O}$ $100^{\circ} \mathrm{C}$

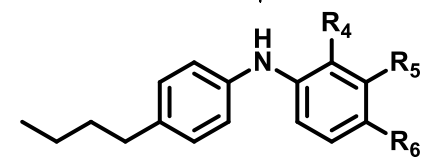
$21 \mathbf{R}_{\mathbf{4}}=\mathrm{COOH}$
$\mathbf{R}_{5}=\mathrm{H}$
$\mathbf{R}_{6}=\mathrm{H}$
$22 \mathbf{R}_{\mathbf{4}}=\mathrm{H}$
$\mathbf{R}_{5}=\mathrm{COOH}$
$\mathbf{R}_{6}=\mathrm{H}$
$23 \quad \mathbf{R}_{4}=\mathrm{H}$
$\mathbf{R}_{\mathbf{5}}=\mathrm{H}$
$\mathbf{R}_{6}=\mathrm{COOH}$

Scheme 3 
Table 2

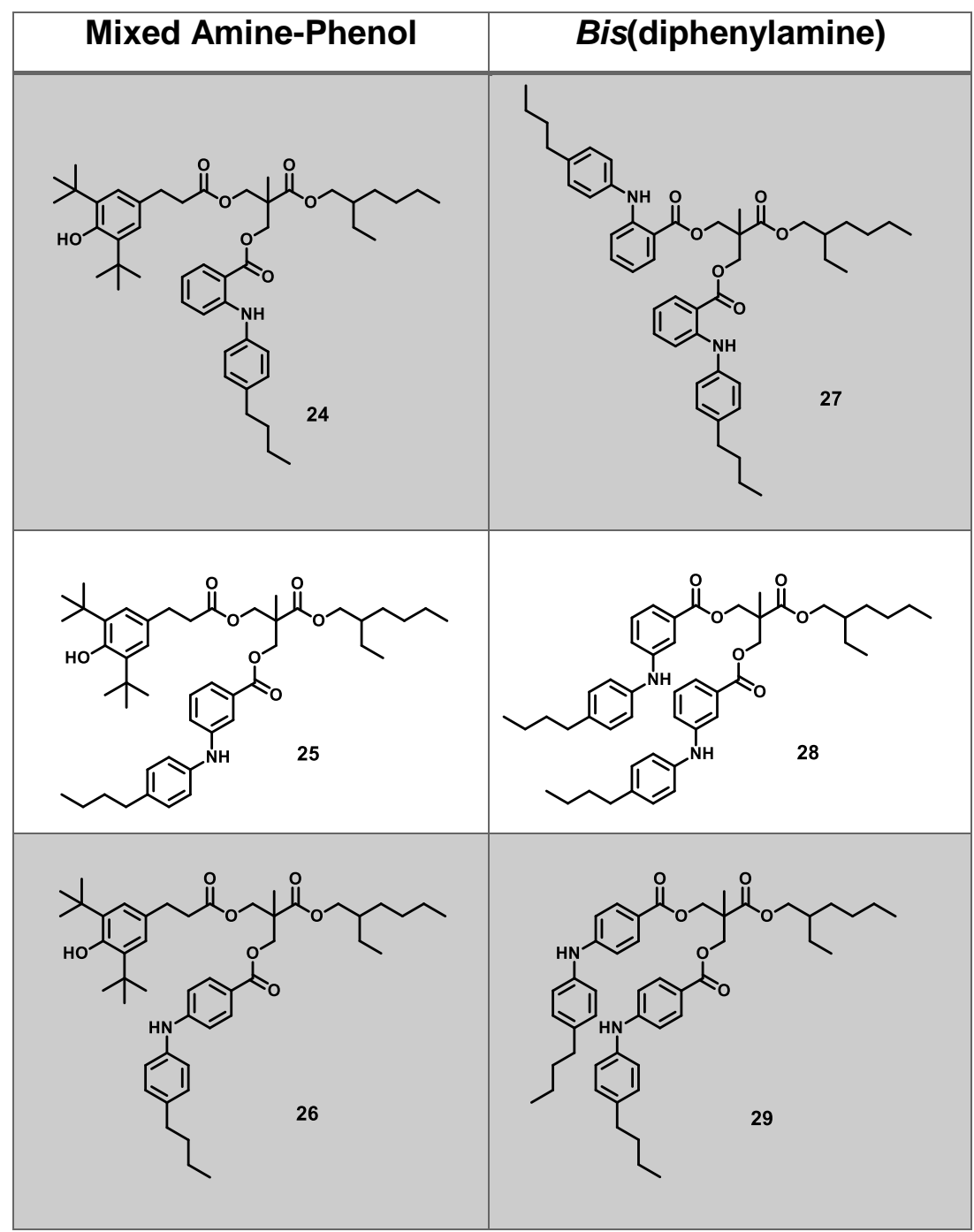




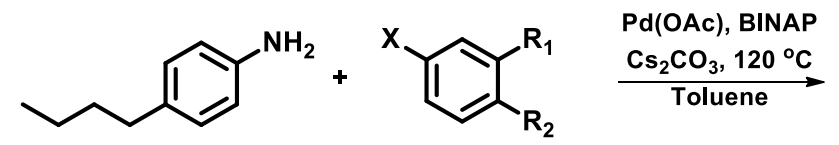

$1631 \quad \mathbf{X}=\mathrm{Br} \quad \mathbf{R}_{\mathbf{1}}=\mathrm{CH}_{2} \mathrm{CH}_{2} \mathrm{COOCH}_{3} \quad \mathbf{R}_{\mathbf{2}}=\mathrm{H}$

$33 \mathbf{X}=\mathrm{Br} \quad \mathbf{R}_{\mathbf{1}}=\mathrm{H} \quad \mathbf{R}_{\mathbf{2}}=\mathrm{CH}_{2} \mathrm{COOCH}_{2} \mathrm{CH}_{3}$

$35 \mathbf{X}=\mathrm{Br} \quad \mathbf{R}_{\mathbf{1}}=\mathrm{H} \quad \mathbf{R}_{\mathbf{2}}=\mathrm{CH}_{2} \mathrm{CH}_{2} \mathrm{COOCH}_{3}$

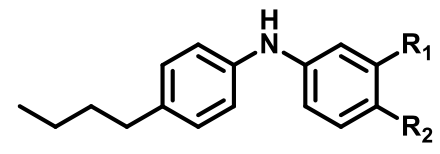

$30 \mathbf{R}_{\mathbf{1}}=\mathrm{CH}_{2} \mathrm{CH}_{2} \mathrm{COOCH}_{3} \mathbf{R}_{\mathbf{2}}=\mathrm{H}$

$32 \quad \mathbf{R}_{\mathbf{1}}=\mathrm{H} \quad \mathbf{R}_{\mathbf{2}}=\mathrm{CH}_{2} \mathrm{COOCH}_{2} \mathrm{CH}_{3}$ $34 \mathbf{R}_{\mathbf{1}}=\mathrm{H} \quad \mathbf{R}_{\mathbf{2}}=\mathrm{CH}_{2} \mathrm{CH}_{2} \mathrm{COOCH}_{3}$

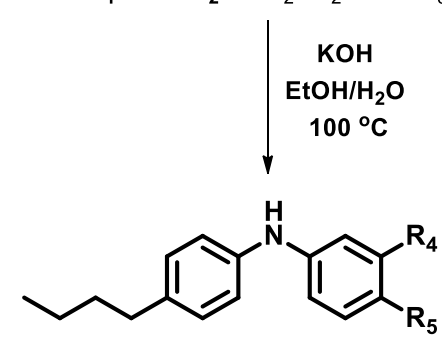

$\begin{array}{lll}36 & \mathbf{R}_{\mathbf{4}}=\mathrm{CH}_{2} \mathrm{CH}_{2} \mathrm{COOH} & \mathbf{R}_{\mathbf{5}}=\mathrm{H} \\ \mathbf{3 7} & \mathbf{R}_{\mathbf{4}}=\mathrm{H} & \mathbf{R}_{\mathbf{5}}=\mathrm{CH}_{2} \mathrm{COOH} \\ \mathbf{3 8} & \mathbf{R}_{\mathbf{4}}=\mathrm{H} & \mathbf{R}_{\mathbf{5}}=\mathrm{CH}_{2} \mathrm{CH}_{2} \mathrm{COOH}\end{array}$

Scheme 4 
Table 3

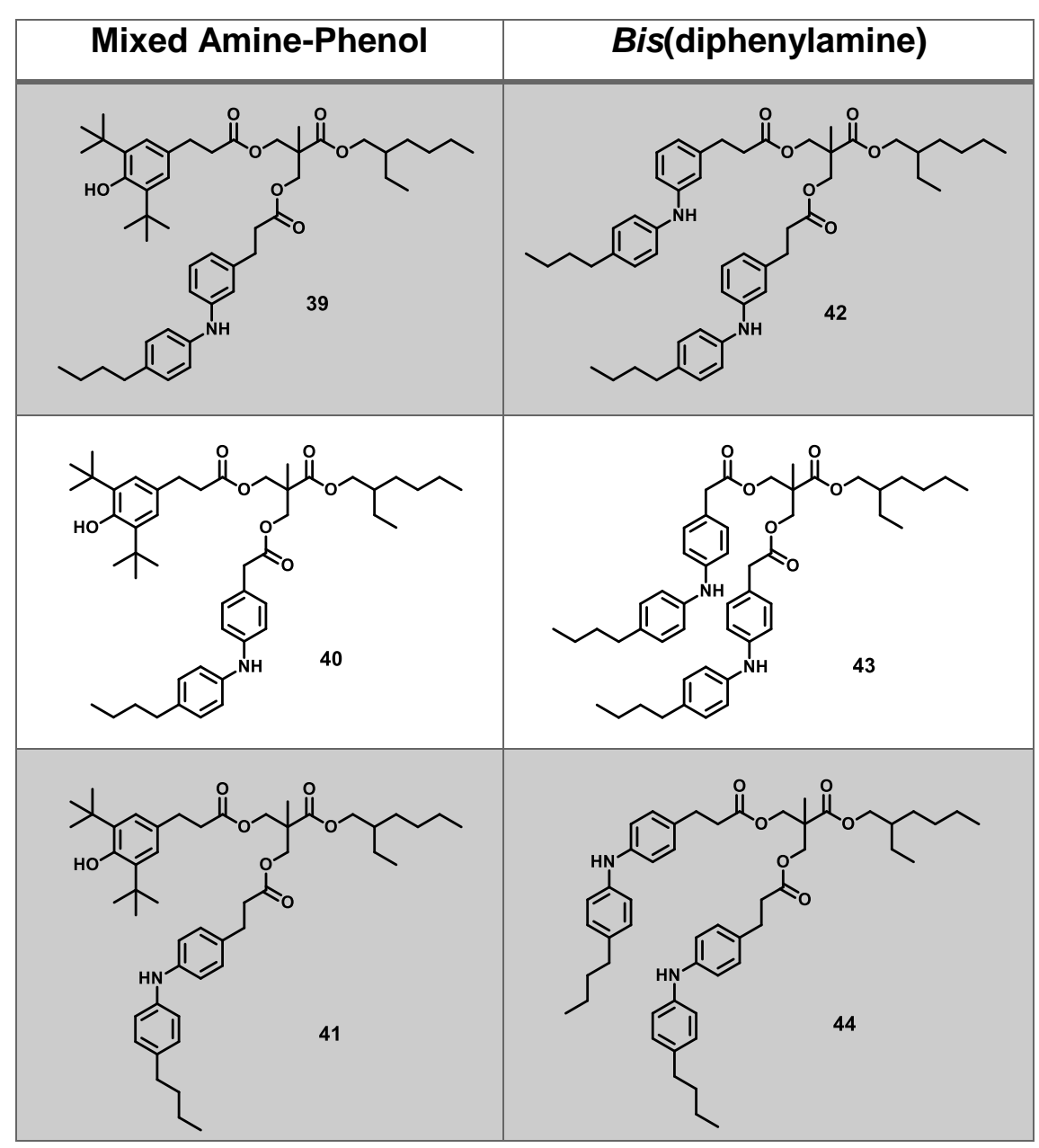




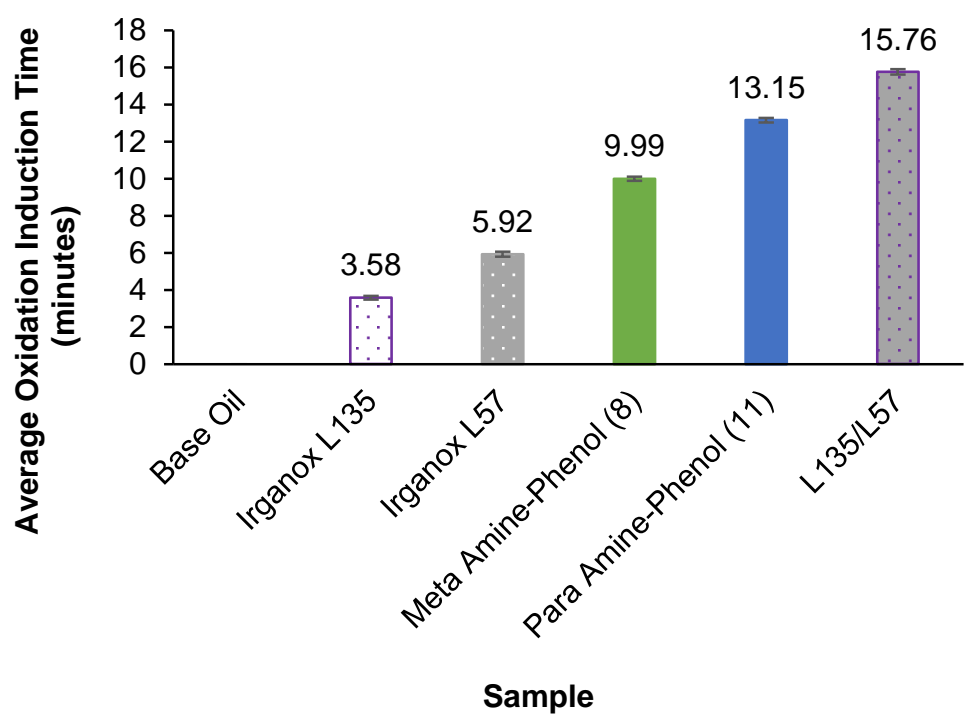

Figure 4

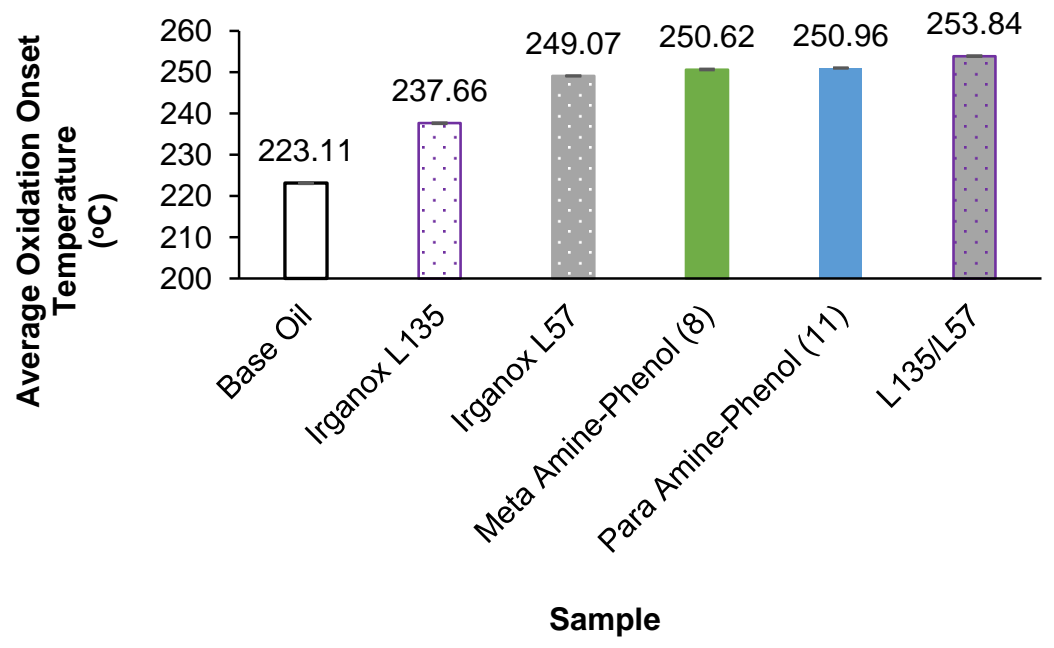

Figure 5 


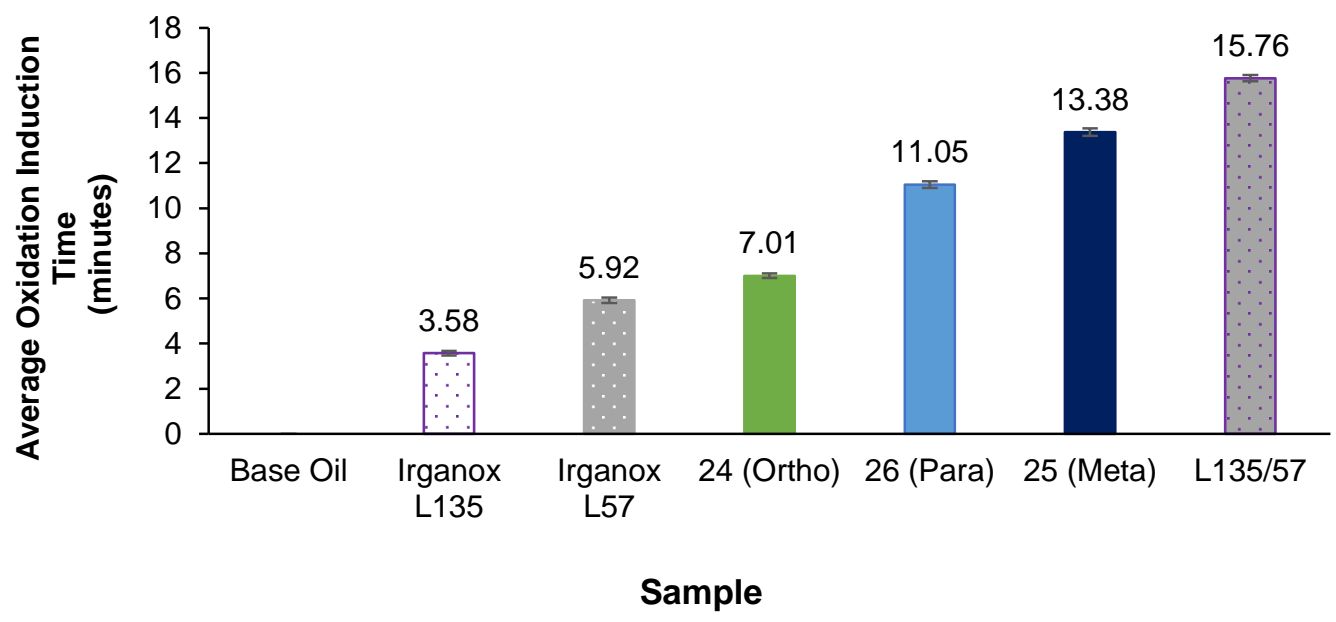

Figure 6

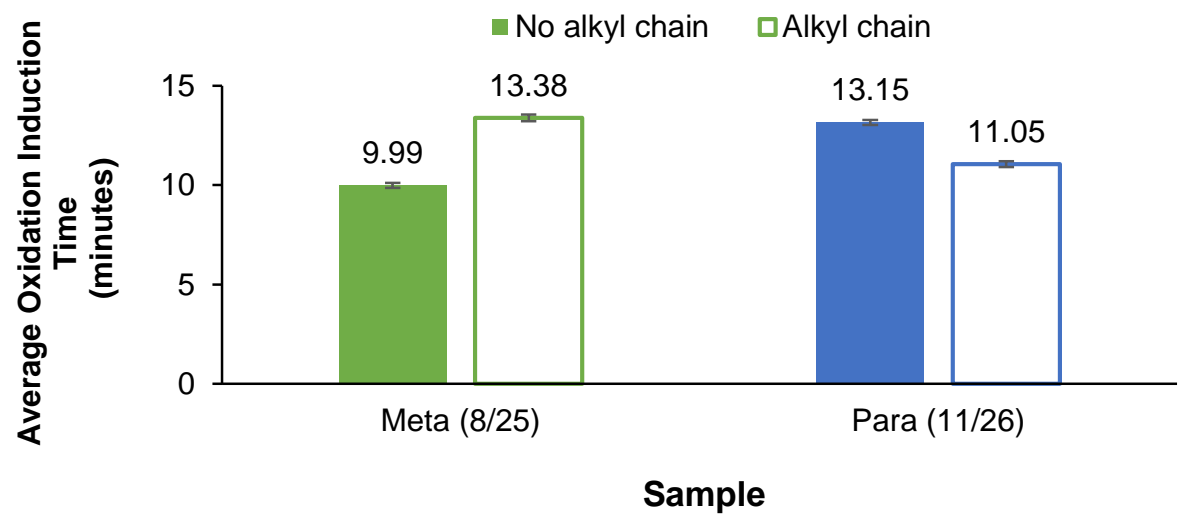

Figure 7 


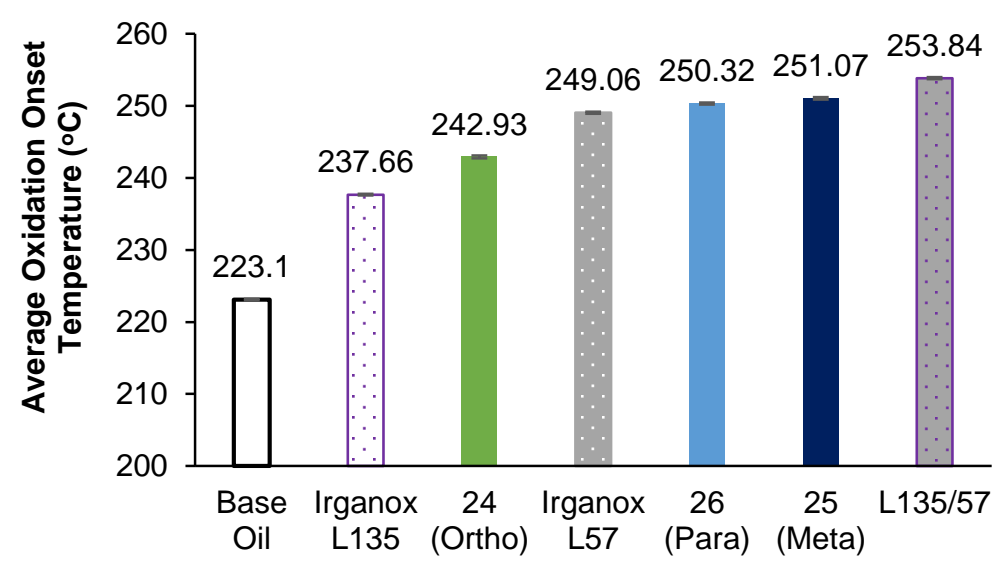

Sample

Figure 8

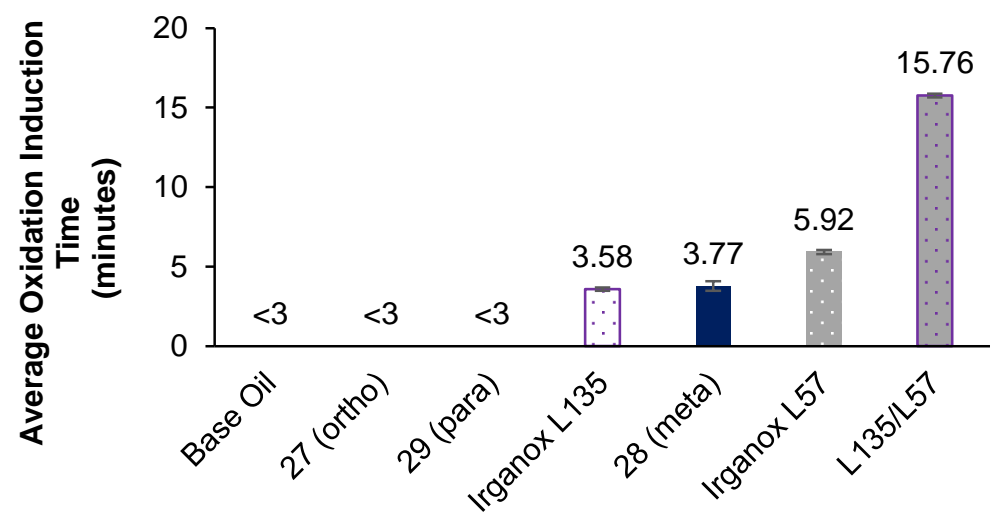

Sample

Figure 9 


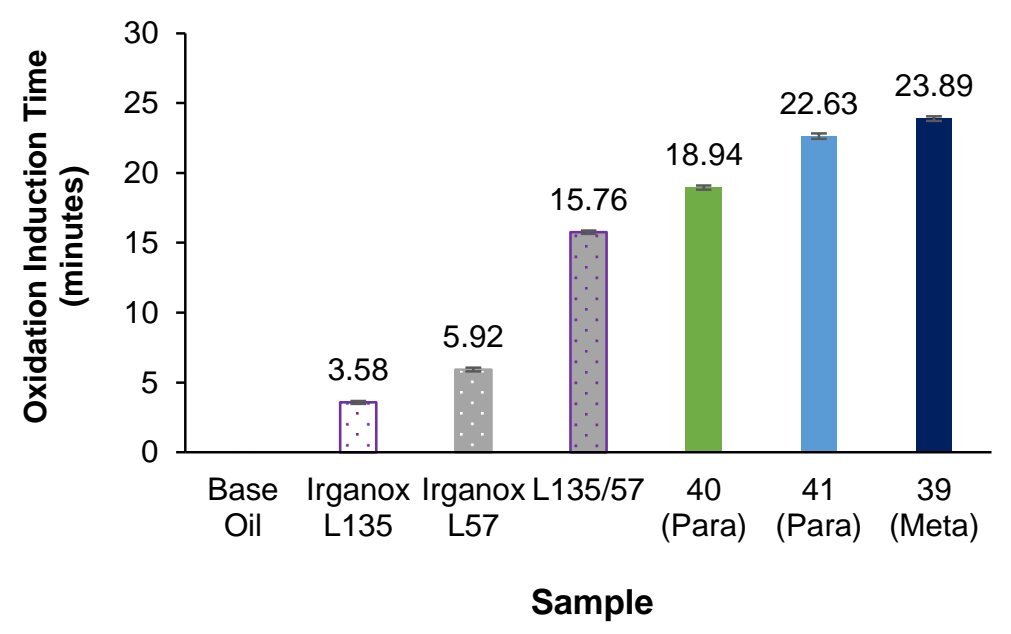

Figure 10

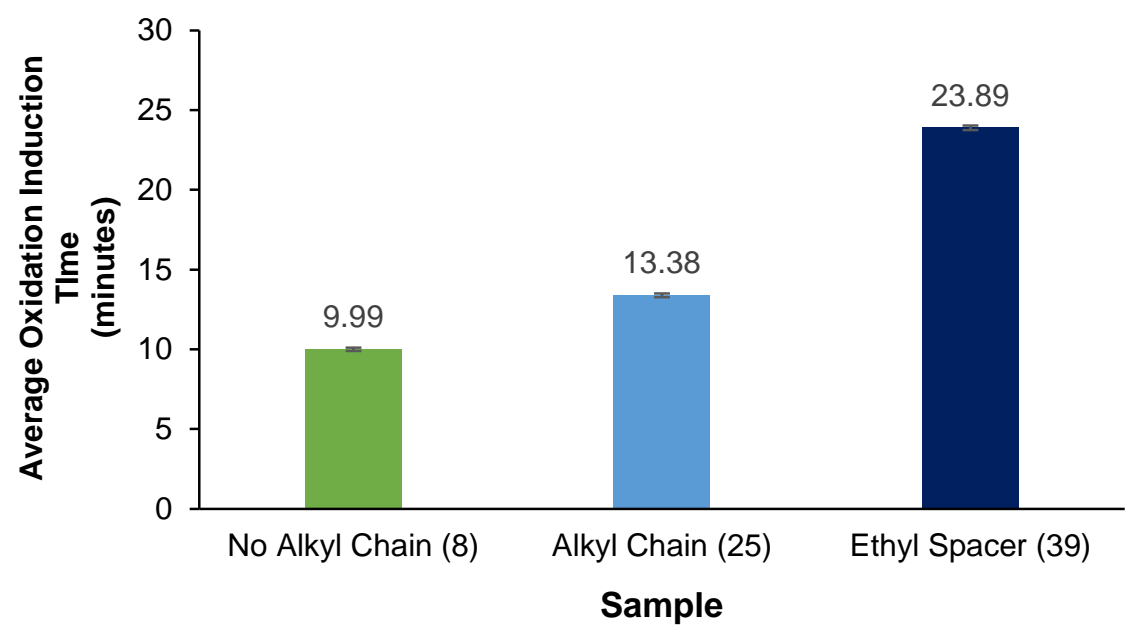

Figure 11 


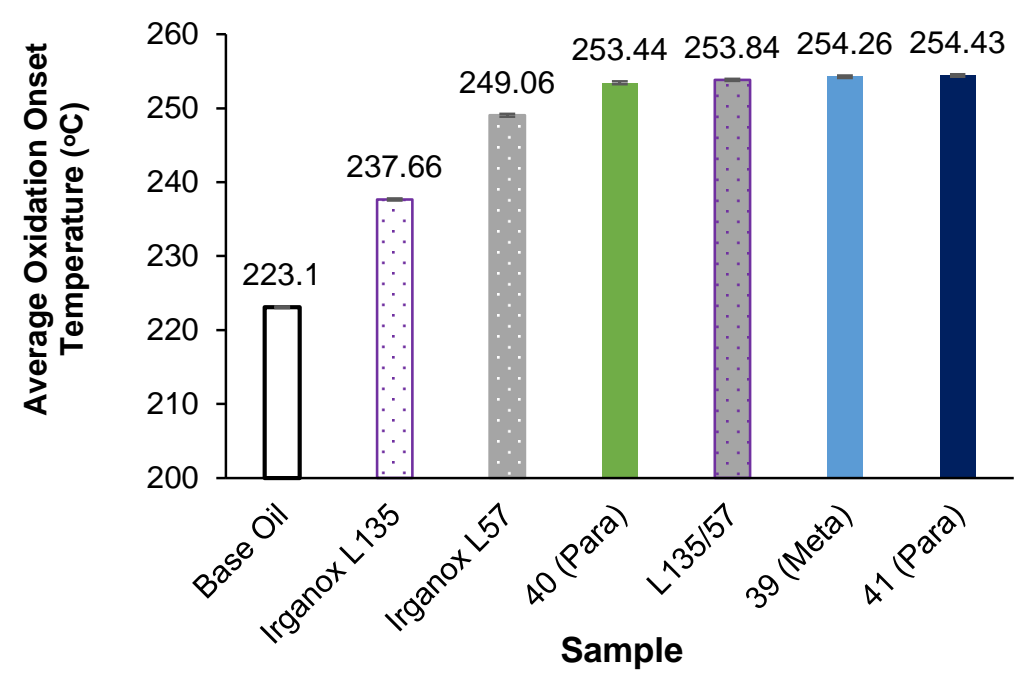

Figure 12 